\title{
Electron temperature fluctuations in planetary nebulae ${ }^{\star}$
}

\author{
A. C. Krabbe and M. V. F. Copetti
}

\begin{abstract}
Laboratório de Análise Numérica e Astrofísica, Departamento de Matemática, Universidade Federal de Santa Maria, 97119-900 Santa Maria, RS, Brazil

e-mail: angela@lana.ccne.ufsm.br
\end{abstract}

Received 20 April 2005 /Accepted 25 July 2005

\begin{abstract}
An observational study of the spatial variation of the electron temperature and density in 10 galactic planetary nebulae is presented. The data consist of long-slit spectra of high signal-to-noise ratio in the 3100 to $6900 \AA$ range. Electron temperatures were determined from the $[\mathrm{O} \operatorname{III}](\lambda 4959+\lambda 5007) / \lambda 4363$ and $[\mathrm{N}$ II $](\lambda 6548+\lambda 6583) / \lambda 5755$ ratios and from the Balmer discontinuity. Electron densities were estimated from the $[\mathrm{S} \mathrm{II}] \lambda 6716 / \lambda 6731$, [Cl III] $\lambda 5517 / \lambda 5537$, and [Ar IV] $\lambda 4711 / \lambda 4740$ ratios. Electron temperature variations of low amplitude were found across the nebular surface in the planetary nebulae studied. The temperature distribution across each nebula presents a variance relative to the mean corresponding to $0.0003 \leq t_{s}^{2}(\mathrm{Bal}) \leq 0.0078,0.0003 \leq t_{s}^{2}(\mathrm{~N}$ II $) \leq 0.0097$, and $0.0011 \leq t_{s}^{2}(\mathrm{O}$ III $) \leq 0.0050$. A systematic spatial variation of electron density has been detected in most of objects (NGC 1535, NGC 2438, NGC 2440, NGC 3132, NGC 3242, NGC 6302, NGC 6563, and NGC 7009). The remaining objects (NGC 6781 and NGC 6853) have not shown any significant electron density dependence on position. NGC 2438, NGC 6563, NGC 6781, and NGC 6853 are in general the most diffuse and probably evolved objects studied here, with low mean densities in the range $N_{\mathrm{e}}(\mathrm{S}$ II $) \approx 95-158 \mathrm{~cm}^{-3}$. An anti-correlation between temperature and density was found for NGC 2438 and NGC 3132, with the electron temperature increasing with the decrease of electron density and a correlation between temperature and density was found for NGC 2440, NGC 3242, NGC 6302, and NGC 7009, with the electron temperature increasing with the increase of electron density. These relationships seem to be associated with the structure of the nebula. The nebulae in which the correlation between temperature and density is present are ring shaped. The anti-correlation between temperature and density is found in bipolar planetary nebulae that are denser in the centre of the nebula.
\end{abstract}

Key words. ISM: planetary nebulae: general

\section{Introduction}

A fundamental issue that still has not been satisfactorily understood in abundance determinations in planetary nebulae and $\mathrm{H}$ II regions is that the abundance of heavy elements derived from their recombination lines are systematically higher than those derived from their collisionally excited emission lines. These discrepancies have been vastly reported in the literature and huge differences have been found. For example, Liu et al. (2000) determined abundances of C, N, O and Ne in the planetary nebula NGC 6153 from recombination lines and found that they are about 10 times higher than those derived from forbidden lines. This difference is even larger, a factor of about 20 times higher, for the galactic bulge planetary nebula M 1-42 (Liu et al. 2001).

One plausible explanation for such discrepancies would be the presence of large internal variation of electron temperature in nebulae. These temperature fluctuations were initially proposed by Peimbert (1967) to explain the considerable differences found between the temperature estimations based on

* Figures 5-23 are only available in electronic form at http://www . edpsciences.org distinct methods in the $\mathrm{H}$ II regions M 8, M 17, and the Orion Nebula. However, high values of temperature fluctuations are required to reconcile the abundances derived from recombination and forbidden lines, which are not predicted by standard photoionization models (Kingdon 1995).

Recently, direct determinations of electron temperature fluctuations have been obtained for the planetary nebulae NGC 4361 (Liu 1998) and NGC 7009 (Rubin et al. 2002), and for the H II regions 30 Doradus (Krabbe \& Copetti 2002) and Orion Nebula (Rubin et al. 2003; O’Dell et al. 2003) from pointto-point measurements of electron temperature and very low temperature fluctuations have been measured. Up to now these four objects are the only ones for which direct estimations of electron temperature fluctuations have been published. So, observational studies of point-to-point measurements of electron temperature in a larger number of objects are of fundamental importance to estimate the amplitude of temperature fluctuations in gaseous nebulae.

This paper reports a study on the spatial variation of electron temperature and density in 10 galactic planetary nebulae. The electron temperature estimates were derived from pointto-point measurements of the $[\mathrm{O}$ III $](\lambda 4959+\lambda 5007) / \lambda 4363$ 
and $[\mathrm{NII}](\lambda 6548+\lambda 6583) / \lambda 5755$ ratios and from the Balmer discontinuity, obtained from long slit spectrophotometry at high signal-to-noise ratio. Point-to-point measurements of electron densities obtained from the [S II] $\lambda 6716 / \lambda 6731$, [Ar IV] $\lambda 4711 / \lambda 4740$, and $[\mathrm{Cl}$ III] $\lambda 5517 / \lambda 5537$ ratios have also been obtained. In a further paper abundances of heavy elements derived from recombination and forbidden lines will be estimated and compared.

\section{Observations and reductions}

The observations were carried out on January, July and December 2002 with the Boller \& Chivens spectrograph attached to the $1.52 \mathrm{~m}$ telescope of the European Southern Observatory (ESO), Chile, and on September 1994 and May 2002 with the Cassegrain spectrograph of the $1.6 \mathrm{~m}$ telescope at the Laboratório Nacional de Astrofísica (LNA), Brazil. We used a Loral CCD of $2688 \times 512$ pixels at ESO and at LNA we used a SITe CCD of $1024 \times 1024$ pixels on May 2002 and an EEV CCD of $800 \times 1024$ pixels on September 1994 . We used a grid of 1200 grooves $\mathrm{mm}^{-1}$ at LNA and at ESO we used a grid of 2400 grooves $\mathrm{mm}^{-1}$ during the January and July runs and a grid of 1200 grooves $\mathrm{mm}^{-1}$ during the December run. The spatial scale was $0.82^{\prime \prime} \mathrm{pxl}^{-1}$ for the Loral CCD, $0.90^{\prime \prime} \mathrm{pxl}^{-1}$ for the EEV CCD, and $1.0^{\prime \prime} \mathrm{pxl}^{-1}$ for the SITe CCD. The slits used have entrances on the plane of sky of $2^{\prime \prime} \times 250^{\prime \prime}$ for the observations at ESO and $2^{\prime \prime} \times 320^{\prime \prime}$ for the observations at LNA. Dome flat-field exposures were taken at the beginning and at the end of the nights. Several bias frames were made along each night. Spectrophotometric standard stars were observed for flux calibration. Spectra of a He-Ar-Ne lamp were taken before and after each object exposure for wavelength calibration.

The objects included in this study are listed in Table 1. Multiple spectra with different exposure times were taken at a same slit position in each nebula to increase the signal-tonoise ratio. The exposures times were limited to $1800 \mathrm{~s}$ to minimize the effects of cosmic rays, and the short exposures of 120 $\mathrm{s}$ or more were taken to measure the intensities of the brightest emission lines, which were close to saturation or saturated in longer exposures. The slit was east-west oriented and centered on the central star in each nebula. Table 2 lists the number and length of the exposures, the dispersion, the spectral resolution, measured as the full-width-at-half-maximum $F W H M$ of the emission lines of comparison lamps, the wavelength range of the spectra, the telescope used and the date of the observations.

The data reduction (bias correction, flat-fielding, cosmic ray cleaning, wavelength and flux calibrations, $1 \mathrm{D}$ spectra extraction) was made using the $I R A F$ software. In order to increase the signal-to-noise ratio, a rebinning of the CCD rows along the spatial direction was performed, giving a sampling of $1.64^{\prime \prime} \mathrm{pxl}^{-1}$.

The line intensities were obtained by integrating the flux over a linear local continuum between two given limits. These measurements were made with the splot routine of the IRAF package. All the line intensities of a given spectrum were normalized to $\mathrm{H} \beta$. The error associated with the line flux intensities were estimated by $\sigma^{2}=\sigma_{\text {cont }}^{2}+\sigma_{\text {line }}^{2}$, where $\sigma_{\text {cont }}$ and $\sigma_{\text {line }}$ are the continuum rms and the Poisson error of the line respectively. The effect of the interstellar extinction was corrected by comparing the $\mathrm{H} \gamma / \mathrm{H} \beta$ and $\mathrm{H} \alpha / \mathrm{H} \beta$ ratios measured in each aperture with the theoretical ones by Hummer (1987) for an electron temperature of $10000 \mathrm{~K}$ and a density of $100 \mathrm{~cm}^{-3}$. The Galactic reddening function of Savage \& Mathis (1979) was used. The final intensity of a given emission line was the average of the line values corrected for interstellar extinction obtained from each spectrum.

The Balmer jump was measured by linearly fitting the observed continua on both sides of the discontinuity. On the blue side of the discontinuity, the continuum is well defined. However, on the red side the spectra were crowded by recombination lines and some extrapolation was required. The Balmer discontinuity, $\left[F_{\lambda}(\lambda 3646-)-F_{\lambda}(\lambda 3646+)\right]$, was normalized to $\mathrm{H} \beta$ using the observed intensity of H11. Due to the small separation between the Balmer discontinuity and the H11 line, the interstellar reddening is negligible. The errors in the Balmer discontinuity were obtained from the errors associated to the linear fittings of the continuum and from the error for the flux of $\mathrm{H} 11$.

\section{Determination of the electron temperature and density}

Electron temperatures were derived from the $[\mathrm{O}$ III $](\lambda 4959+$ $\lambda 5007) / \lambda 4363$ and $[\mathrm{NII}](\lambda 6548+\lambda 6583) / \lambda 5755$ intensity ratios and electron densities from the $[\mathrm{S}$ II] $\lambda 6716 / \lambda 6731$, $[\mathrm{Cl} \mathrm{III}] \lambda 5517 / \lambda 5537$, and $[\mathrm{Ar} \mathrm{IV}] \lambda 4711 / \lambda 4740$ intensity ratios by solving numerically the equilibrium equations for a $n$ level atom using the temden routine of the nebular package of the STSDAS/IRAF. The references for the collision strengths C, transition probabilities T, and energy levels $\mathrm{E}$ used are listed in Table 3. For some planetary nebulae electron temperatures were also calculated from the ratio of the nebular Balmer discontinuity to $\mathrm{H} \beta,\left[F_{\lambda}(\lambda 3646-)-F_{\lambda}(\lambda 3646+)\right] / F(\mathrm{H} \beta)$ by interpolation of the values presented by Osterbrock (1989).

There are several factors that can increase the errors in the measurement of electron density. The most significant factor is the saturation of line ratios at both low and high values of electron density. Stanghellini \& Kaler (1989) consider that the measurements of electron density from [S II], [Cl III] and [Ar IV] ratios are reliable in the ranges $2.45<\log N_{\mathrm{e}}\left(\mathrm{cm}^{-3}\right)<3.85,3<$ $\log N_{\mathrm{e}}\left(\mathrm{cm}^{-3}\right)<4.95$ and $3.3<\log N_{\mathrm{e}}\left(\mathrm{cm}^{-3}\right)<5.55$, respectively. Other source of error for electron density determined from the $[\mathrm{Ar}$ IV] $\lambda 4740 / \lambda 4711$ ratio is that the $[\mathrm{Ar}$ IV] $\lambda 4711$ is blended with $\mathrm{He} \mathrm{I} \lambda$ 4713. We discounted the helium contribution to this blend by assuming the intensity of the $\mathrm{He} I \lambda 4713$ is one-tenth of the intensity of He I $\lambda 4471$ (Benjamin et al. 1999). The dependence of the electron density $N_{\mathrm{e}}$ on the assumed electron temperature $T_{\mathrm{e}}$ is another source of error. For the range of electron temperatures found in our sample of objects, the errors in the determinations of electron densities due to the assumption of a constant electron temperature in each nebula are below $5 \%$, with exceptions of few density measurements with errors of the order of up to $20 \%$.

In the determination of electron temperature there are many potential sources of uncertainty. For the electron temperatures 
Table 1. Selected objects.

\begin{tabular}{|c|c|c|c|c|c|c|c|}
\hline Object & $\alpha(2000)$ & $\delta(2000)$ & $l$ & $b$ & $d(\mathrm{pc})$ & $\theta\left({ }^{\prime \prime}\right)$ & Other names \\
\hline NGC 1535 & $04^{\mathrm{h}} 14^{\mathrm{m}} 15^{\mathrm{s}} .6$ & $-12^{\mathrm{h}} 44^{\mathrm{m}} 22^{\mathrm{s}} .5$ & $206^{\circ} .48$ & $-40^{\circ} .56$ & $\begin{array}{l}1649[2] \\
2140[1] \\
2283[3]\end{array}$ & $9.2[2]$ & PK 206-40 1 \\
\hline NGC 2438 & $07 \quad 4150.3$ & -144408.8 & 231.80 & 4.12 & $\begin{array}{r}1519[2] \\
850[1] \\
1203[3]\end{array}$ & $35.2[2]$ & PK 231+04 2 \\
\hline NGC 2440 & $07 \quad 4155.3$ & -181230.5 & 234.84 & 2.42 & $\begin{array}{l}1088[2] \\
1010[1] \\
1348[3]\end{array}$ & 16.4 [2] & PK 234+02 1 \\
\hline NGC 3132 & 100701.7 & -402611.7 & 272.11 & 12.40 & $\begin{array}{l}1079[2] \\
1110[1] \\
1251[3]\end{array}$ & $28.0[2]$ & PK 272+12 1 \\
\hline NGC 3242 & 102446.0 & -183832.3 & 261.05 & 32.05 & $\begin{array}{r}810[2] \\
860[1] \\
1083[3]\end{array}$ & $20.2[2]$ & PK $261+321$, Ghost of Jupiter Nebula \\
\hline NGC 6302 & 171344.6 & -370611.7 & 349.51 & 1.06 & $\begin{array}{l}415[2] \\
950[1] \\
525[3]\end{array}$ & $22.3[2]$ & PK 349+01 1, Bug Nebula, Gum 60 \\
\hline NGC 6563 & 181202.5 & -335206.0 & 358.50 & -7.34 & $\begin{array}{l}1867[2] \\
1270[1] \\
1631[3]\end{array}$ & $22.6[2]$ & PK 358-07 1 \\
\hline NGC 6781 & 191828.2 & 063223.0 & 41.84 & -2.99 & $\begin{array}{l}904[2] \\
910[1] \\
699[3]\end{array}$ & $35.0[2]$ & PK 041-02 1 \\
\hline NGC 6853 & 195936.2 & 224315.6 & 60.84 & -3.70 & $\begin{array}{l}400[2] \\
250[1] \\
262[1]\end{array}$ & $100.0[2]$ & M 27, PK 060-03 1, Dumbbell Nebula \\
\hline NGC 7009 & 210410.8 & -112148.0 & 37.76 & -34.57 & $\begin{array}{r}871[2] \\
1280[1] \\
1201[3]\end{array}$ & $13.4[2]$ & PK 037-34 1, Saturn Nebula \\
\hline
\end{tabular}

References: [1] Cahn \& Kaler (1971); [2] Maciel \& Pottasch (1980); [3] Cahn et al. (1992).

Conventions: $\alpha, \delta$ : equatorial coordinates; $l, b$ : galactic coordinates, in degree; $d$ : distance; $\theta$ : angular radius.

Catalogues: Gum = Gum (1955); PK = Perek \& Kohoutek (1967).

estimated from the Balmer discontinuity the main difficulty lies in determining the continuum redward of the discontinuity, which is crowded by Balmer lines. Hence, the intensity of the continuum must be measured at longer wavelengths and extrapolated to $\lambda 3646+$. For the electron temperatures measurements from the $[\mathrm{O} \operatorname{III}](\lambda 4959+$ $\lambda 5007) / \lambda 4363$ and $[\mathrm{N} I I](\lambda 6548+\lambda 6583) / \lambda 5755$ ratios the greatest problem lies in the estimation of the flux of the $[\mathrm{O} I I I] \lambda 4363$ and $[\mathrm{NII}] \lambda 5755$ lines, which are very weak comparing with the other two lines of each ratio. However, this uncertainty is attenuated by the high signalto-noise ratio of the data presented in this paper. An other source of error for electron temperature estimated from the $[\mathrm{N}$ II $](\lambda 6548+\lambda 6583) / \lambda 5755$ ratio is the contribution to the flux of the auroral line $[\mathrm{N}$ II] $\lambda 5755$ due to recombination. The contribution reduces the electron temperature. For our sample of objects, it is only significant in the central regions of NGC 7009. We have estimated the recombination contribution to the intensity of the $[\mathrm{NII}] \lambda 5755$ line using the equation $I_{\mathrm{R}}(\lambda 5755) / I(\mathrm{H} \beta)=3.19\left(T_{\mathrm{e}} / 10^{4} \mathrm{~K}\right)^{0.30} \times \mathrm{N}^{++} / \mathrm{H}^{+}$given by Liu et al. (2000), assuming $\mathrm{N}^{++} / \mathrm{H}^{+}=3.10 \times 10^{-4}$ (Liu et al. 1995) for the central aperture and scaling this value by the flux in N II 5676 for other apertures. For the range of variation of the electron density found in the planetary nebulae studied the dependence of the electron temperature estimate on the assume electron density is practically insignificant, with exception of NGC 6302, in which high densities of the order of $15000 \mathrm{~cm}^{-3}$ have been derived for the central region. The procedure to derive temperatures and densities from forbidden line ratios was as follows. We calculated the electron densities $N_{\mathrm{e}}(\mathrm{S} \mathrm{II}), N_{\mathrm{e}}\left(\mathrm{Cl}\right.$ III), and $N_{\mathrm{e}}(\mathrm{Ar} \mathrm{IV})$ assuming an initial electron temperature of $10000 \mathrm{~K}$. Then, a mean density for each nebula was used to derive the electron temperatures $T_{\mathrm{e}}(\mathrm{O}$ III $)$ and $T_{\mathrm{e}}(\mathrm{N}$ II). Finally the electron densities were recalculated adopting mean values of electron temperature for each nebula. For NGC 6302, the $T_{\mathrm{e}}(\mathrm{N}$ II $)$ estimates are sensitive to the assume densities in the central region of nebula. Therefore, for this object different value of electron density were adopted for different apertures.

\section{Results}

Figures 1 and 2 present a sample of spectra from areas with different surface brightness showing the $[\mathrm{N}$ II $] \lambda 4363$ and 
Table 2. Journal of observations.

\begin{tabular}{|c|c|c|c|c|c|c|}
\hline Object & Date & Telescope & $\Delta \lambda(\AA)$ & $F W H M(\AA)$ & Disp. $\left(\AA \mathrm{pxl}^{-1}\right)$ & Exp. time (s) \\
\hline \multirow[t]{2}{*}{ NGC 1535} & 12.30 .2002 & $1.52 \mathrm{~m} \mathrm{ESO}$ & $4630-6940$ & 3.0 & 1.0 & $3 \times 1200+4 \times 300$ \\
\hline & 12.31 .2002 & $1.52 \mathrm{~m}$ ESO & $3120-5430$ & 3.0 & 1.0 & $3 \times 1200+3 \times 120$ \\
\hline \multirow[t]{5}{*}{ NGC 2438} & 01.04 .2002 & $1.52 \mathrm{~m}$ ESO & $3840-5100$ & 1.5 & 0.50 & $4 \times 1500$ \\
\hline & 01.05 .2002 & $1.52 \mathrm{~m} \mathrm{ESO}$ & $3840-5100$ & 1.5 & 0.50 & $4 \times 600$ \\
\hline & 05.02 .2002 & $1.60 \mathrm{~m} \mathrm{LNA}$ & $6120-6900$ & 2.8 & 0.75 & $3 \times 1200$ \\
\hline & 12.30 .2002 & $1.52 \mathrm{~m} \mathrm{ESO}$ & $4630-6940$ & 3.0 & 1.0 & $3 \times 1200$ \\
\hline & 12.31 .2002 & $1.52 \mathrm{~m}$ ESO & $3120-5430$ & 3.0 & 1.0 & $3 \times 1200$ \\
\hline \multirow[t]{4}{*}{ NGC 2440} & 01.05 .2002 & $1.52 \mathrm{~m} \mathrm{ESO}$ & $3840-5100$ & 1.5 & 0.50 & $4 \times 1500+4 \times 120$ \\
\hline & 05.02 .2002 & 1.60 m LNA & $6120-6900$ & 2.8 & 0.75 & $4 \times 600$ \\
\hline & 12.29 .2002 & $1.52 \mathrm{~m} \mathrm{ESO}$ & $3120-5430$ & 3.0 & 1.0 & $3 \times 1500+6 \times 120$ \\
\hline & 12.30 .2002 & $1.52 \mathrm{~m}$ ESO & $4630-6940$ & 3.0 & 1.0 & $2 \times 600+6 \times 100$ \\
\hline \multirow[t]{2}{*}{ NGC 3132} & 12.31 .2002 & $1.52 \mathrm{~m} \mathrm{ESO}$ & $3120-5430$ & 3.0 & 1.0 & $3 \times 600$ \\
\hline & 12.31 .2002 & $1.52 \mathrm{~m} \mathrm{ESO}$ & $4630-6940$ & 3.0 & 1.0 & $1 \times 1200+1 \times 100$ \\
\hline \multirow{6}{*}{ NGC 3242} & 05.02 .2002 & $1.60 \mathrm{~m}$ LNA & $6120-6900$ & 2.8 & 0.75 & $1 \times 300+2 \times 1200$ \\
\hline & 01.04 .2002 & $1.52 \mathrm{~m}$ ESO & $3840-5100$ & 1.5 & 0.50 & $2 \times 600+3 \times 120$ \\
\hline & 01.05 .2002 & $1.52 \mathrm{~m}$ ESO & $3840-5100$ & 1.5 & 0.50 & $3 \times 600$ \\
\hline & 07.10 .2002 & $1.52 \mathrm{~m} \mathrm{ESO}$ & $3300-5100$ & 3.0 & 0.75 & $4 \times 1200$ \\
\hline & 07.10 .2002 & $1.52 \mathrm{~m}$ ESO & $4460-6800$ & 3.0 & 0.75 & $3 \times 600$ \\
\hline & 07.09 .2002 & $1.52 \mathrm{~m} \mathrm{ESO}$ & $4490-6860$ & 3.0 & 0.75 & $6 \times 300+1 \times 120$ \\
\hline \multirow[t]{2}{*}{ NGC 6302} & 07.09 .2002 & $1.52 \mathrm{~m}$ ESO & $4490-6860$ & 3.0 & 0.75 & $4 \times 1800+4 \times 150$ \\
\hline & 07.10 .2002 & $1.52 \mathrm{~m} \mathrm{ESO}$ & $3300-5100$ & 3.0 & 0.75 & $3 \times 600$ \\
\hline NGC 6563 & 07.10 .2002 & $1.52 \mathrm{~m}$ ESO & $4460-6800$ & 3.0 & 0.75 & $3 \times 1200$ \\
\hline NGC 6781 & 07.09 .2002 & $1.52 \mathrm{~m} \mathrm{ESO}$ & $4690-6860$ & 3.0 & 0.75 & $2 \times 1200$ \\
\hline \multirow[t]{2}{*}{ NGC 6853} & 07.09 .2002 & $1.52 \mathrm{~m}$ ESO & $4490-6860$ & 3.0 & 0.75 & $3 \times 1200$ \\
\hline & 07.10 .2002 & $1.52 \mathrm{~m}$ ESO & $4460-6800$ & 3.0 & 0.75 & $2 \times 1200$ \\
\hline \multirow[t]{5}{*}{ NGC 7009} & 09.12 .1994 & $1.60 \mathrm{~m} \mathrm{LNA}$ & $4100-5030$ & 2.8 & 1.0 & $9 \times 120+8 \times 600$ \\
\hline & 09.12.1994 & $1.60 \mathrm{~m}$ LNA & $6200-7000$ & 2.8 & 1.0 & $9 \times 120+2 \times 600$ \\
\hline & 07.09.2002 & $1.52 \mathrm{~m} \mathrm{ESO}$ & $4490-6860$ & 3.0 & 0.75 & $15 \times 120$ \\
\hline & 07.10 .2002 & $1.52 \mathrm{~m}$ ESO & $4460-6800$ & 3.0 & 0.75 & $2 \times 1200+2 \times 600$ \\
\hline & 07.10 .2002 & $1.52 \mathrm{~m}$ ESO & $3300-5100$ & 3.0 & 0.75 & $3 \times 1200$ \\
\hline
\end{tabular}

Table 3. Line ratios and references for the atomic data.

\begin{tabular}{llll}
\hline \hline Line ratios & T & C & E \\
\hline$[\mathrm{O}$ III $](\lambda 4959+\lambda 5007) / \lambda 4363$ & {$[6]$} & {$[2]$} & {$[1,8]$} \\
{$[\mathrm{N}$ II $](\lambda 6548+\lambda 6583) / \lambda 5755$} & {$[6]$} & {$[2]$} & {$[1,9]$} \\
{$\left[\mathrm{S} \mathrm{II}^{\prime} \lambda 6716 / \lambda 6731\right.$} & {$[7,10]$} & {$[3]$} & {$[1,10]$} \\
{$\left[\mathrm{Cl}_{\mathrm{III}}\right] \lambda 5517 / \lambda 5537$} & {$[11,12]$} & {$[4]$} & {$[1]$} \\
{$[$ Ar IV $] \lambda 4711 / \lambda 4740$} & {$[12,13]$} & {$[5]$} & {$[1]$} \\
\hline
\end{tabular}

References: [1] Bowen (1960); [2] Lennon \& Burke (1994); [3] Ramsbottom et al. (1996); [4] Butler \& Zeippen (1989); [5] Zeippen et al. (1987), [6] Wiese et al. (1996); [7] Keenan et al. (1993); [8] Moore (1985); [9] Williams \& Livio (1995); [10] Verner et al. (1996); [11] Mendoza (1983); [12] Kaufman \& Sugar (1986); [13] Mendoza \& Zeippen (1982).

[N II] 45755 lines for NGC 6302 and NGC 2440. Figure 3 shows the Balmer discontinuity and our fitting of the observed continua for NGC 7009. Spatial profiles along the slit of the $\mathrm{H} \beta$ flux, electron temperature and electron density are shown in Figs. 6-23. Table 4 presents some statistics of the electron temperature and density measurements, including the number $N$ of distinct nebular areas, the median, the first and the third quartiles, $Q 1$ and $Q 3$ respectively (limits between which $50 \%$ of the values lie), the minimum and maximum, and the mean and the standard deviation $\sigma$ weighted by the flux in $\mathrm{H} \beta$. Next, the observed objects are discussed separately.

\subsection{NGC 1535}

NGC 1535 is a bright planetary nebula with a pronounced ring structure of about $20^{\prime \prime} \times 17^{\prime \prime}$ within a fainter, larger disk of about $48^{\prime \prime} \times 42^{\prime \prime}$ (Perek \& Kohoutek 1967). This object shows a rich spectrum of rather high excitation. The intensities of emission lines of low ionization species such as those of [S II], $[\mathrm{Cl} \mathrm{III}]$, and [N II] were not measurable in our spectra. The electron densities were estimated only by the [Ar IV] $\lambda 4711 / \lambda 4740$ ratio, a density indicator for more internal zones of nebulae. A mean value of $N_{\mathrm{e}}(\mathrm{ArIV})=2049 \pm 166 \mathrm{~cm}^{-3}$ was estimated, which is consistent with measurements by other authors (Minkowski \& Aller 1956; Aller \& Walker 1965; Gutierrez-Moreno et al. 1985). The data obtained for NGC 1535 (Figs. 4 and 5) show low amplitude variations of electron density and temperature with the position along the radius of the nebula. We have found that $T_{\mathrm{e}}(\mathrm{O}$ III $)$ are systematically higher than $T_{\mathrm{e}}(\mathrm{Bal})$, with mean values of $T_{\mathrm{e}}(\mathrm{O}$ III $)=$ $12607 \pm 148 \mathrm{~K}$ and $T_{\mathrm{e}}(\mathrm{Bal})=10131 \pm 105 \mathrm{~K}$. This discrepancy between $T_{\mathrm{e}}(\mathrm{O}$ III $)$ and $T_{\mathrm{e}}(\mathrm{Bal})$ was also found by other authors 
Table 4. Electron temperature and density statistics.

\begin{tabular}{|c|c|c|c|c|c|c|c|c|}
\hline & $N$ & $\min$ & $Q 1$ & median & $Q 3$ & $\max$ & mean & $\sigma$ \\
\hline \multicolumn{9}{|c|}{ NGC 1535} \\
\hline$N_{\mathrm{e}}(\mathrm{Ar} \mathrm{IV})\left(\mathrm{cm}^{-3}\right)$ & 24 & 580 & 1425 & 1671 & 2430 & 3722 & 2049 & 813 \\
\hline$T_{\mathrm{e}}(\mathrm{O}$ III $)(\mathrm{K})$ & 29 & 11672 & 12018 & 12349 & 12709 & 15489 & 12607 & 799 \\
\hline$T_{\mathrm{e}}(\mathrm{Bal})(\mathrm{K})$ & 9 & 9810 & 9743 & 9923 & 10371 & 10560 & 10131 & 315 \\
\hline \multicolumn{9}{|c|}{ NGC 2438} \\
\hline$N_{\mathrm{e}}(\mathrm{S}$ II $)\left(\mathrm{cm}^{-3}\right)$ & 49 & 19 & 98 & 155 & 209 & 340 & 158 & 64 \\
\hline$T_{\mathrm{e}}(\mathrm{N}$ II $)(\mathrm{K})$ & 32 & 10265 & 10843 & 11173 & 11461 & 12265 & 11245 & 490 \\
\hline$T_{\mathrm{e}}(\mathrm{O}$ III $)(\mathrm{K})$ & 45 & 10662 & 11026 & 11590 & 12373 & 14080 & 11541 & 840 \\
\hline \multicolumn{9}{|c|}{ NGC 2440} \\
\hline$N_{\mathrm{e}}(\mathrm{S}$ II $)\left(\mathrm{cm}^{-3}\right)$ & 35 & 256 & 458 & 806 & 1891 & 3360 & 2551 & 1670 \\
\hline$N_{\mathrm{e}}(\mathrm{Cl} \mathrm{III})\left(\mathrm{cm}^{-3}\right)$ & 14 & 487 & 2426 & 3131 & 5276 & 5477 & 4131 & 1809 \\
\hline$N_{\mathrm{e}}(\operatorname{Ar} \mathrm{IV})\left(\mathrm{cm}^{-3}\right)$ & 25 & 0 & 1032 & 1572 & 3031 & 4186 & 3192 & 1694 \\
\hline$T_{\mathrm{e}}(\mathrm{N}$ II $)(\mathrm{K})$ & 35 & 9493 & 10692 & 11822 & 12491 & 14086 & 11838 & 1152 \\
\hline$T_{\mathrm{e}}(\mathrm{O}$ III $)(\mathrm{K})$ & 43 & 13084 & 13612 & 13623 & 14673 & 19291 & 14398 & 1302 \\
\hline$T_{\mathrm{e}}(\mathrm{Bal})(\mathrm{K})$ & 12 & 11178 & 11929 & 14323 & 14993 & 18886 & 13984 & 2218 \\
\hline \multicolumn{9}{|c|}{ NGC 3132} \\
\hline$N_{\mathrm{e}}\left(\mathrm{S}_{\mathrm{II}}\right)\left(\mathrm{cm}^{-3}\right)$ & 37 & 110 & 335 & 558 & 714 & 910 & 617 & 239 \\
\hline$N_{\mathrm{e}}(\mathrm{Cl} \mathrm{III})\left(\mathrm{cm}^{-3}\right)$ & 15 & 953 & 1372 & 1870 & 2891 & 4980 & 2045 & 1138 \\
\hline$N_{\mathrm{e}}(\mathrm{Ar} \mathrm{IV})\left(\mathrm{cm}^{-3}\right)$ & 15 & 399 & 1266 & 1867 & 2333 & 5751 & 2278 & 1533 \\
\hline$T_{\mathrm{e}}(\mathrm{N}$ II $)(\mathrm{K})$ & 30 & 9577 & 9987 & 10357 & 10749 & 12456 & 10163 & 726 \\
\hline$T_{\mathrm{e}}(\mathrm{O}$ III $)(\mathrm{K})$ & 24 & 9411 & 9413 & 9682 & 10325 & 12421 & 9889 & 721 \\
\hline \multicolumn{9}{|c|}{ NGC 3242} \\
\hline$N_{\mathrm{e}}(\mathrm{S}$ II $)\left(\mathrm{cm}^{-3}\right)$ & 13 & 1150 & 2332 & 2818 & 3845 & 7643 & 3081 & 1640 \\
\hline$N_{\mathrm{e}}(\mathrm{Cl} \mathrm{III})\left(\mathrm{cm}^{-3}\right)$ & 19 & 201 & 1381 & 1822 & 3183 & 11602 & 3188 & 2757 \\
\hline$N_{\mathrm{e}}(\operatorname{Ar} \mathrm{IV})\left(\mathrm{cm}^{-3}\right)$ & 29 & 576 & 1851 & 2629 & 3525 & 4413 & 3247 & 1156 \\
\hline$T_{\mathrm{e}}(\mathrm{O}$ III $)(\mathrm{K})$ & 35 & 11201 & 11565 & 12252 & 12881 & 16792 & 12213 & 1378 \\
\hline$T_{\mathrm{e}}(\mathrm{Bal})(\mathrm{K})$ & 24 & 8055 & 8907 & 9692 & 9864 & 10150 & 9541 & 652 \\
\hline \multicolumn{9}{|c|}{ NGC 6302} \\
\hline$N_{\mathrm{e}}(\mathrm{S} \mathrm{II})\left(\mathrm{cm}^{-3}\right)$ & 91 & 11 & 238 & 797 & 1669 & 14509 & 6811 & 5846 \\
\hline$N_{\mathrm{e}}(\mathrm{Cl} \mathrm{III})\left(\mathrm{cm}^{-3}\right)$ & 43 & 362 & 2977 & 7468 & 15890 & 130192 & 37694 & 34280 \\
\hline$N_{\mathrm{e}}(\operatorname{Ar} \mathrm{IV})\left(\mathrm{cm}^{-3}\right)$ & 51 & 88 & 2283 & 4590 & 8121 & 15939 & 10238 & 6178 \\
\hline$T_{\mathrm{e}}(\mathrm{N}$ II $)(\mathrm{K})$ & 94 & 11437 & 12001 & 12428 & 13016 & 17771 & 13798 & 1580 \\
\hline$T_{\mathrm{e}}(\mathrm{O}$ III $)(\mathrm{K})$ & 80 & 15009 & 16443 & 17071 & 17889 & 20461 & 18357 & 1596 \\
\hline \multicolumn{9}{|c|}{ NGC 6563} \\
\hline$N_{\mathrm{e}}\left(\mathrm{S}_{\mathrm{II}}\right)\left(\mathrm{cm}^{-3}\right)$ & 47 & 1 & 70 & 142 & 176 & 267 & 134 & 70 \\
\hline$T_{\mathrm{e}}(\mathrm{N}$ II $)(\mathrm{K})$ & 43 & 9835 & 10522 & 10731 & 11059 & 12421 & 10743 & 471 \\
\hline \multicolumn{9}{|c|}{ NGC 6781} \\
\hline$N_{\mathrm{e}}\left(\mathrm{S}_{\mathrm{II}}\right)\left(\mathrm{cm}^{-3}\right)$ & 57 & 1 & 40 & 131 & 233 & 343 & 135 & 94 \\
\hline$T_{\mathrm{e}}(\mathrm{N}$ II $)(\mathrm{K})$ & 31 & 9208 & 10391 & 10913 & 11386 & 12927 & 10763 & 781 \\
\hline \multicolumn{9}{|c|}{ NGC 6853} \\
\hline$N_{\mathrm{e}}(\mathrm{S}$ II $)\left(\mathrm{cm}^{-3}\right)$ & 126 & 4 & 40 & 88 & 131 & 553 & 95 & 80 \\
\hline$T_{\mathrm{e}}(\mathrm{N}$ II $)(\mathrm{K})$ & 142 & 9488 & 10450 & 10832 & 11146 & 12629 & 10898 & 572 \\
\hline \multicolumn{9}{|c|}{ NGC 7009} \\
\hline$N_{\mathrm{e}}(\mathrm{S}$ II $)\left(\mathrm{cm}^{-3}\right)$ & 35 & 253 & 806 & 2352 & 3152 & 3598 & 3164 & 1683 \\
\hline$N_{\mathrm{e}}(\mathrm{Cl} \mathrm{III})\left(\mathrm{cm}^{-3}\right)$ & 19 & 1793 & 4980 & 5363 & 7294 & 7909 & 6239 & 1610 \\
\hline$N_{\mathrm{e}}(\operatorname{ArIV})\left(\mathrm{cm}^{-3}\right)$ & 13 & 471 & 891 & 2735 & 7448 & 9930 & 4314 & 3143 \\
\hline$T_{\mathrm{e}}(\mathrm{N}$ II $)(\mathrm{K})$ & 17 & 9847 & 10477 & 10749 & 11524 & 12487 & 11163 & 785 \\
\hline$T_{\mathrm{e}}(\mathrm{O}$ III $)(\mathrm{K})$ & 27 & 8534 & 9503 & 9856 & 10184 & 11740 & 10117 & 761 \\
\hline$T_{\mathrm{e}}(\mathrm{Bal})(\mathrm{K})$ & 21 & 7340 & 8250 & 8521 & 8740 & 9045 & 8589 & 500 \\
\hline
\end{tabular}




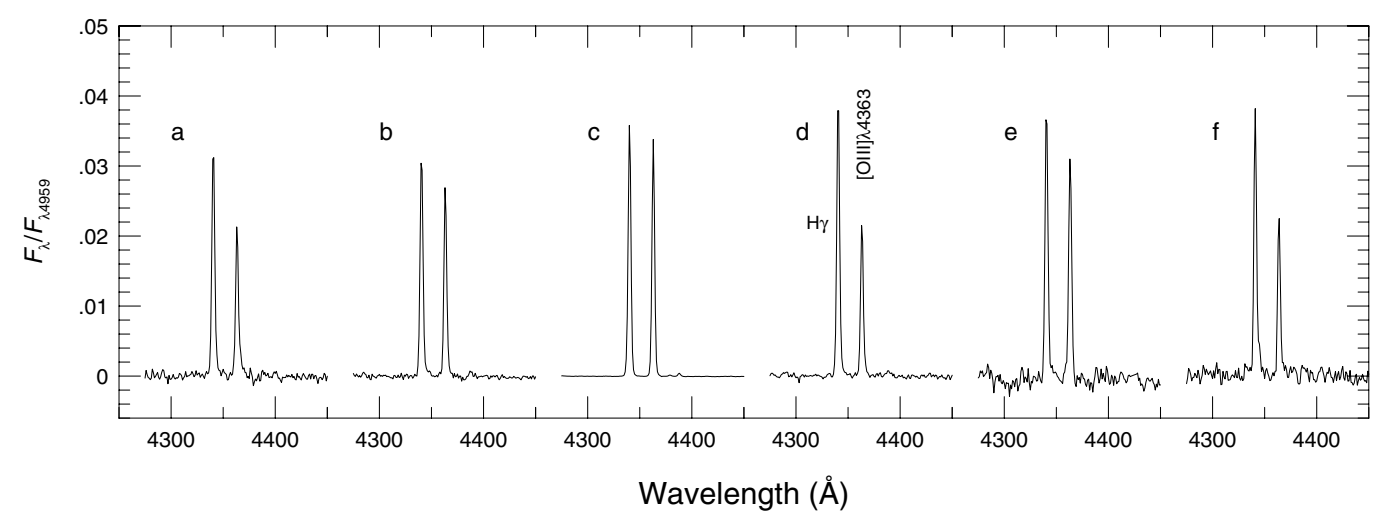

Fig. 1. A sample of spectra in the range of 4275 to $4460 \AA$ from areas with different surface brightnesses for NGC 6302 . The corresponding positions are marked in Fig. 15. To emphasize the variation of the $[\mathrm{O} I I I](\lambda 4959+\lambda 5007) / \lambda 4363$ ratio, the flux scale was normalized to the peak of $[\mathrm{O}$ III] $] \lambda 4959$.

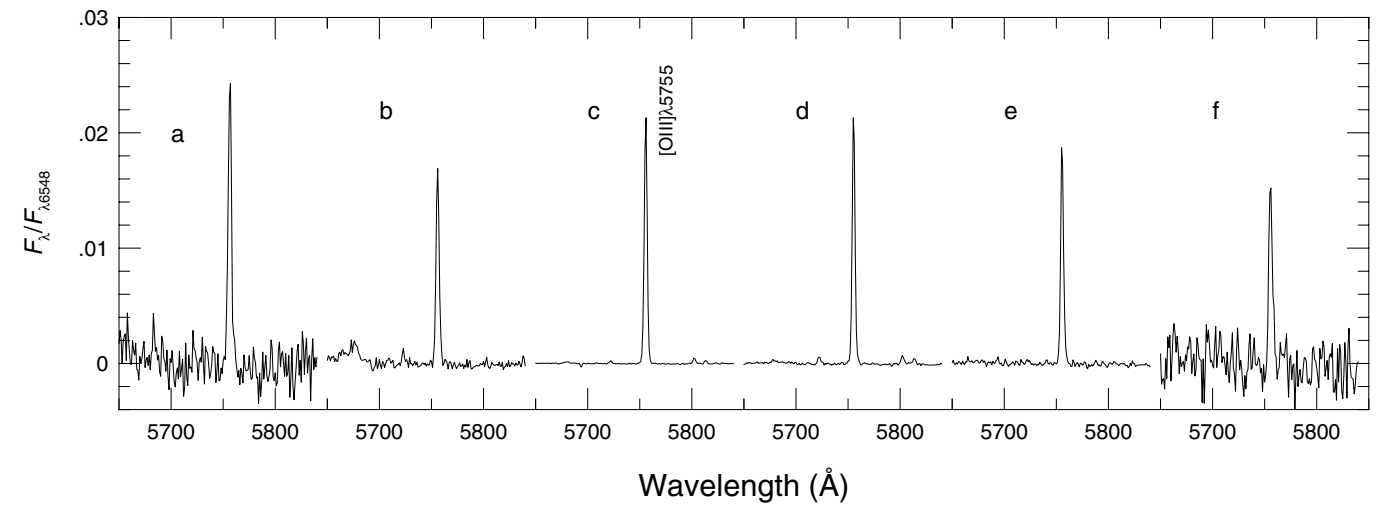

Fig. 2. A sample of spectra in the range of 5650 to $5850 \AA$ from areas with different surface brightnesses for NGC 2440 . The corresponding positions are marked in Fig. 9. To emphasize the variation of the $[\mathrm{N} I I](\lambda 6548+\lambda 6583) / \lambda 5755$ ratio, the flux scale was normalized to the peak of [N II] $\lambda 6548$.

in other planetary nebulae and was attributed to temperature fluctuations ranging on average from $t^{2}=0.03$ to $t^{2}=0.07$ (Peimbert 1971; Liu \& Danziger 1993; Zhang et al. 2004).

\subsection{NGC 2438}

This planetary nebula exhibits a bright, round inner ring and a detached faint halo. Figure 6 shows a very smooth variation of density with a weighted mean value of $N_{\mathrm{e}}(\mathrm{S}$ II $)=158 \pm 9 \mathrm{~cm}^{-3}$. The low electron densities found in NGC 2438 are characteristic of objects in a more advanced stage of nebular evolution. This interpretation was given by Guerrero \& Manchado (1999) and is in agreement with the dynamical age of around $17000 \mathrm{yr}$ derived from an expansion velocity of $22 \mathrm{~km} \mathrm{~s}^{-1}$ (Meatheringham et al. 1988) and a distance of $2.0 \mathrm{kpc}$ (Pottasch 1983).

The electron temperatures estimated from the $[\mathrm{O} \operatorname{III}](\lambda 4959+\lambda 5007) / \lambda 4363$ and $[\mathrm{N}$ II $](\lambda 6548+$ $\lambda 6584) / \lambda 5755$ ratios (see Fig. 7) present variations of small amplitude along the slit. The mean electron temperatures derived from these two line intensity ratios are in good agreement. We found weighted mean electron temperatures of $T_{\mathrm{e}}(\mathrm{O}$ III $)=11541 \pm 125 \mathrm{~K}$ and $T_{\mathrm{e}}(\mathrm{N}$ II $)=11245 \pm 87 \mathrm{~K}$.
The spatial variation of the temperature $T_{\mathrm{e}}(\mathrm{O}$ III) has a behavior opposite to the profile of the surface nebular brightness, with the surface brightness in $\mathrm{H} \beta$ decreasing with the increase of electron temperature. This was also found in 30 Doradus (Krabbe \& Copetti 2002). A possible interpretation of such behavior is that it might be indicating an anti-correlation between [O III] temperature and density. In fact, such anti-correlation, although somewhat subtle, can be seen in our data for NGC 2438 , with the density increasing and electron temperature decreasing in the direction of the areas with higher surface brightness.

\subsection{NGC 2440}

NGC 2440 is a bipolar planetary nebula that may be taken as the prototype of Peimbert type I (Peimbert 1978), an object rich in $\mathrm{He}$ and $\mathrm{N}$. It is a bright, somewhat elongated object which shows two distinct central condensations with fainter outer filaments and it has a wide variety of surface brightness and excitation. The density profiles obtained for NGC 2440 from the [S II], [Ar IV], and [Cl III] ratios (see Fig. 8) show a well defined spatial gradient, with the density decreasing from the center to the edges. The densities derived from these three ratios show similar variations across the nebular surface. We have 


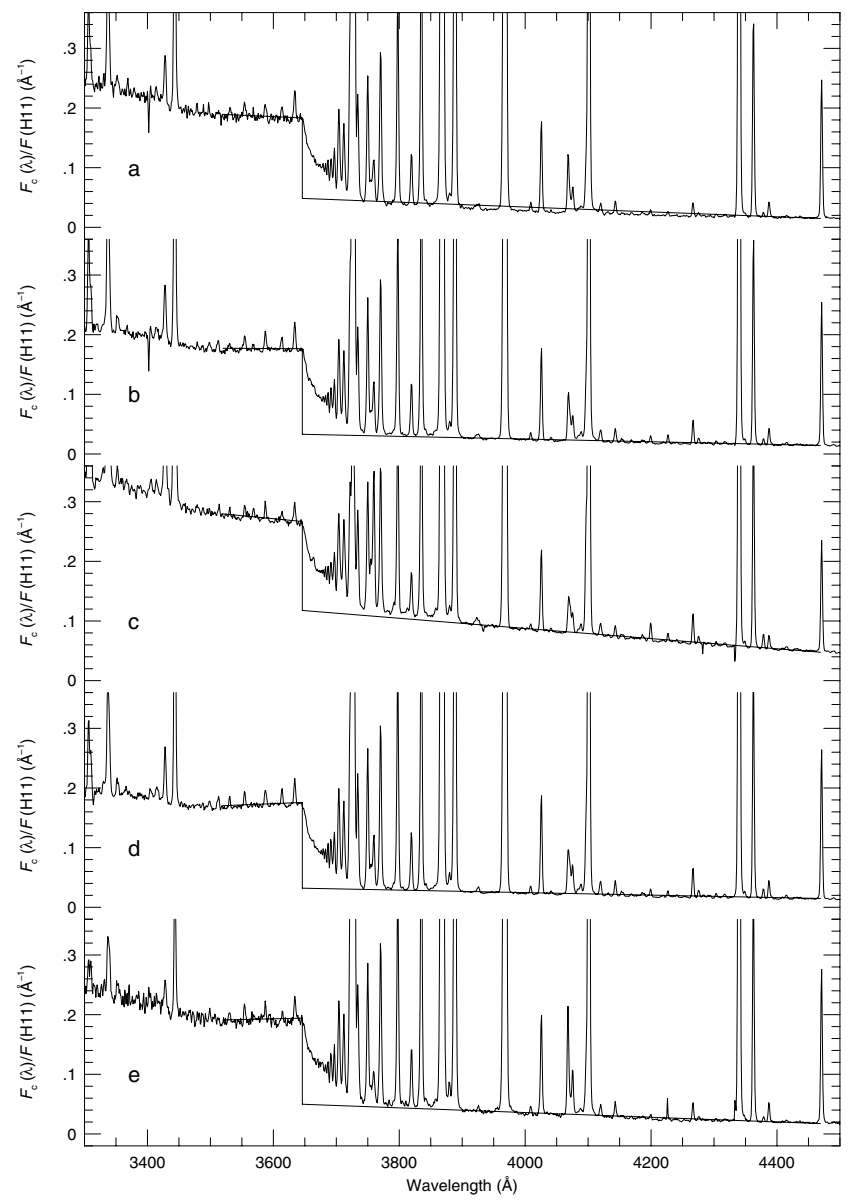

Fig. 3. A sample of spectra showing the observed Balmer discontinuity from different surface brightnesses for NGC 7009. The corresponding positions are marked in Fig 9. The scale of flux was normalized to the flux of H11.

derived mean electron densities of $N_{\mathrm{e}}(\mathrm{S} \mathrm{II})=2551 \pm 282 \mathrm{~cm}^{-3}$, $N_{\mathrm{e}}(\mathrm{Cl}$ III $)=4131 \pm 483 \mathrm{~cm}^{-3}$, and $N_{\mathrm{e}}(\mathrm{ArIV})=3192 \pm$ $339 \mathrm{~cm}^{-3}$. Our mean electron densities are systematically lower than the ones derived by Shields et al. (1981). We have recalculated the electron densities from their [S II], [Ar IV], and [Cl III] ratios using the same atomic parameters and electron density adopted in this paper, and we have derived values from about 1000 to $2000 \mathrm{~cm}^{-3}$ higher than those from our own data. However, these authors have concentrated their observations on the nebular core, where the values of electron density are systematically higher.

As can be seen in the Fig. 9, the electron temperatures derived from the [O III] and $[\mathrm{N} \mathrm{II}]$ ratios and from the observed Balmer discontinuity show a systematic variation across the nebular surface. The electron temperatures derived from the $[\mathrm{NII}]$ and $[\mathrm{O} I I I]$ ratio are decreasing from the centre to the edges in the area of the nebula with higher surface brightness in $\mathrm{H} \beta$ and increasing in the direction of the outskirts of the nebula, where the surface brightness is lower. At the same time, the profile of Balmer temperature is completely different of the profiles of [N II] and [O III] temperatures, not showing any relation with the surface brightness of the nebula. For the entire set of apertures observed we found weighted

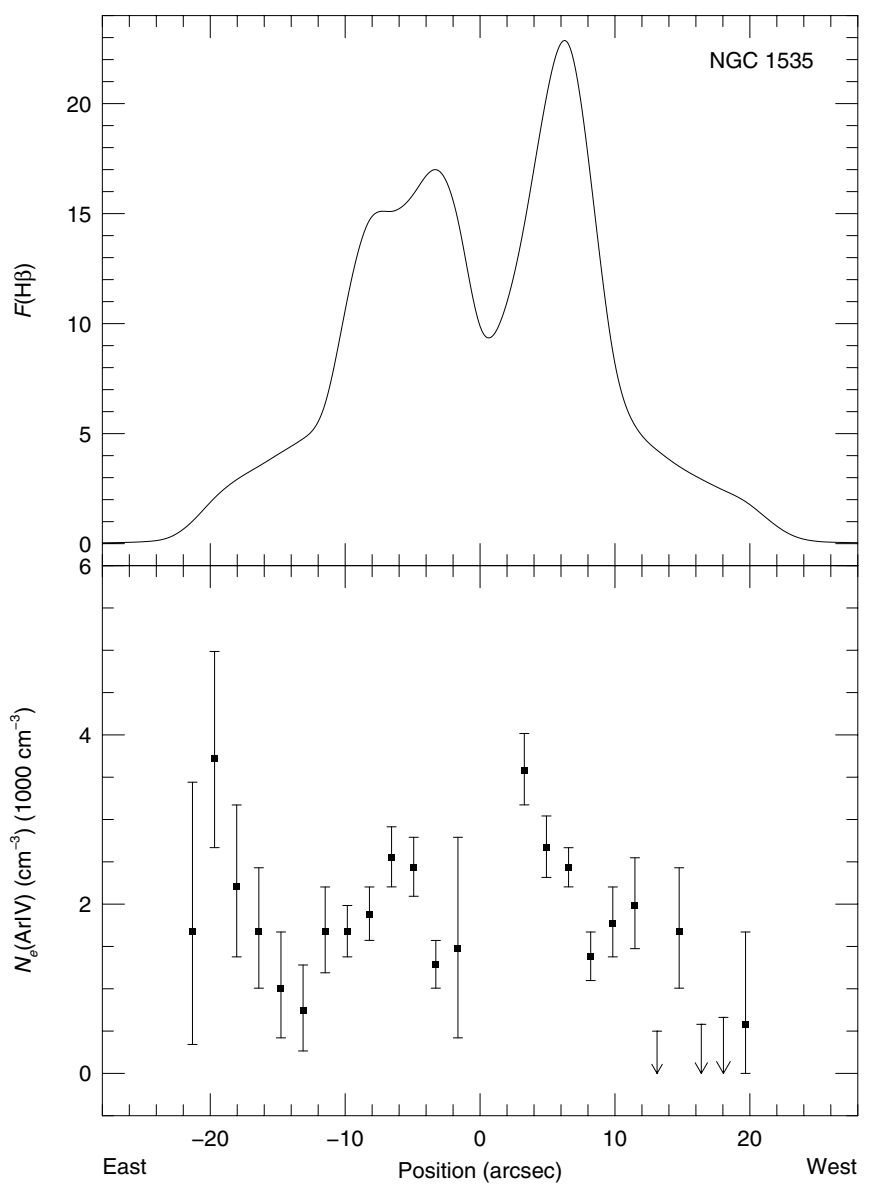

Fig. 4. NGC 1535. Spatial profiles of $\mathrm{H} \beta$ flux (in units of $\left.10^{-14} \mathrm{erg} \mathrm{cm}^{-2} \mathrm{~s}^{-1}\right)$ and $N_{\mathrm{e}}(\operatorname{Ar} \mathrm{IV})$.

mean electron temperatures of $T_{\mathrm{e}}(\mathrm{O}$ III $)=14398 \pm 199 \mathrm{~K}$, $T_{\mathrm{e}}(\mathrm{N}$ II $)=11838 \pm 195 \mathrm{~K}$, and $T_{\mathrm{e}}(\mathrm{Bal})=13984 \pm 640 \mathrm{~K}$. The [O III] temperatures are in good agreement with those obtained by Hyung \& Aller (1998), Liu \& Danziger (1993), Peimbert \& Torres-Peimbert (1987), Gutierrez-Moreno et al. (1985) and Shields et al. (1981); however the mean electron temperature derived for the [N II] zone by these authors are about 1000 to $2000 \mathrm{~K}$ lower than the ones obtained from our own observations. On the other hand, our estimations are compatible with the ones of $T_{\mathrm{e}}(\mathrm{N}$ II $)=11500 \mathrm{~K}$ and $T_{\mathrm{e}}(\mathrm{NII})=13800 \mathrm{~K}$ found in two regions of NGC 2440 by Boeshaar (1974). If these higher temperatures are an artifact of the line flux estimation it will be certainly due to the errors in the intensities of the [N II] $\lambda 5755$ line, since weak emission lines are biased towards overestimated values (Rola \& Pelat 1994). However, our intensities of [N II] $\lambda 5755$ line have high signal-to-noise ratio and therefore the errors associated with these measurements are not considerable.

Higher electron temperatures have been estimated from other indicators. Rowlands et al. (1989) derived electron temperatures of $17100 \mathrm{~K}$ using the $[\mathrm{Ne} \mathrm{V}](\lambda 24.3 \mu \mathrm{m} / \lambda 3426 \AA)$ ratio and $22000 \mathrm{~K}$ using the $[\mathrm{O} \operatorname{IV}](\lambda 25.9 \mu \mathrm{m} / \lambda 1400 \AA)$ ratio. Although the temperature estimates derived from $[\mathrm{NeV}](\lambda 24.3 \mu \mathrm{m} / \lambda 3426 \AA)$ are quite dependent on electron 
density and hence more susceptible to uncertainties, these high temperatures are an indication of the presence of considerably hotter zones in the inner part of the nebula.

\subsection{NGC 3132}

This is a moderately high excitation planetary nebula showing an elliptical structure. As for NGC 2440, the nitrogen is enhanced in the ionized material and it can be placed in the Peimbert type I category (Peimbert 1978). The results for this object are illustrated in Figs. 10 and 11. Due to the presence of a bright central star some emission lines from the central region of the nebula were more susceptible to uncertainties or could not be measured. A well defined spatial variation of the [S II] density has been observed in this object with two density peaks of $N_{\mathrm{e}}(\mathrm{S} \mathrm{II})=910_{-35}^{+38} \mathrm{~cm}^{-3}$ and $N_{\mathrm{e}}(\mathrm{S} \mathrm{II})=874_{-34}^{+36} \mathrm{~cm}^{-3}$ along the east-west direction. The position of the density peak on the east side is shifted for about $3^{\prime \prime}$ to the east of the $\mathrm{H} \beta$ peak and the density peak on the west side is about $1^{\prime \prime}$ away from the $\mathrm{H} \beta$ peak on the west. The [S II] densities seem to have a similar density variation of NGC 2438 , that is, the electron density is increasing with the increase of surface brightness in $\mathrm{H} \beta$, although this is not so clear in the central part, where the measurements are more doubtful. Juguet et al. (1988) have determined densities from the $[\mathrm{S}$ II] $\lambda 6716 / \lambda 6731$ ratio and they have found a double peaked distribution along the north-south direction, reaching up to $N_{\mathrm{e}}(\mathrm{S} \mathrm{II})=1300 \mathrm{~cm}^{-3}$ at the outer region and decreasing to $N_{\mathrm{e}}(\mathrm{S} \mathrm{II})=300 \mathrm{~cm}^{-3}$ at the central position. Unfortunately, for the [Ar IV] and [Cl III] densities it is not possible to observe a variation similar to that of the [S II] densities, probably because there are few measurements of [Ar IV] and [Cl III] densities along the nebular surface. We have derived mean densities of $N_{\mathrm{e}}(\mathrm{Cl}$ III $)=2045 \pm 304 \mathrm{~cm}^{-3}$ and $N_{\mathrm{e}}(\mathrm{Ar} \mathrm{IV})=2278 \pm 425 \mathrm{~cm}^{-3}$, which are about $1500 \mathrm{~cm}^{-3}$ higher than the $[\mathrm{S}$ II] mean density.

The profiles of [N II] and [O III] temperatures shows smooth variations across the nebula. Although it was not possible to estimate the electron temperatures in the central region of nebula, the $[\mathrm{NII}]$ and [O III] temperatures seem to present profiles similar to that of the [O III] temperature in NGC 2438 and the same anti-correlation between temperature and surface nebular brightness. The derived mean temperatures of $T_{\mathrm{e}}(\mathrm{N}$ II $)=10163 \pm 133 \mathrm{~K}$ and $T_{\mathrm{e}}(\mathrm{O}$ III $)=9889 \pm 147 \mathrm{~K}$ are compatible with the estimates of Torres-Peimbert \& Peimbert (1977) and Baessgen \& Grewing (1990).

\subsection{NGC 3242}

NGC 3242 is a high excitation nebula showing a bipolar structure in the central region with a fainter outer halo. Figures 12 and 13 show the results obtained for this nebula. The densities obtained from the $[\mathrm{S}$ II $] \lambda 6716 / \lambda 6731$ and $[\mathrm{Cl} \mathrm{III}] \lambda 5517 / \lambda 5537$ intensity ratios are relatively homogeneous, with mean values of $N_{\mathrm{e}}(\mathrm{S}$ II $)=3081 \pm 455 \mathrm{~cm}^{-3}$ and $N_{\mathrm{e}}(\mathrm{Cl} \mathrm{III})=3188 \pm 632 \mathrm{~cm}^{-3}$. However, the uniformity of the density distribution may be apparent to the fact that the $[\mathrm{Cl} \mathrm{III}]$ and $[\mathrm{S} \mathrm{II}]$ lines are somewhat faint, so the derived density values are rather uncertain. Densities derived from the [Ar IV] $\lambda 4711 / \lambda 4740$ ratio present a smooth variation increasing as the surface brightness $\mathrm{H} \beta$ increases. The densities inferred from [Cl III], [S II], and [Ar IV] emission lines are compatible, indicating that the density is not correlated with the ionization state.

The distribution of electron temperatures estimated from the [O III] ratio and from the observed Balmer discontinuity exhibits variations of very low amplitude across the nebula. Fig. 13 shows that the temperatures obtained from [O III] ratio are systematically higher than those inferred from the observed Balmer discontinuity. We have found mean temperatures of $T_{\mathrm{e}}(\mathrm{O}$ III $)=12213 \pm 233 \mathrm{~K}$ and $T_{\mathrm{e}}(\mathrm{Bal})=9541 \pm 133 \mathrm{~K}$, which agree well with the values measured by Liu \& Danziger (1993). Again, the [O III] temperature tends to increase as the surface brightness in $\mathrm{H} \beta$ increases at the brightest parts of the nebula. Higher temperatures are also found at the outskirts of the nebula.

\subsection{NGC 6302}

This planetary nebula is one of the brightest known in our galaxy. Indeed it is the object in our sample with the highest surface brightness. The optical image of NGC 6302 shows an impressive bipolar morphology. Such nebula has been classified as type $\mathrm{I}$ in the scheme of Peimbert \& Torres-Peimbert (1983), with abundances even more extreme than typical type I nebulae (Aller et al. 1981). Figure 14 reveals a systematic variation with the position in the nebula of the electron densities obtained from the [S II] $\lambda 6716 / \lambda 6731$, [Ar IV] $\lambda 4711 / \lambda 4740$, and $[\mathrm{Cl}$ III $] \lambda 5517 / \lambda 5537$ ratios. The densities derived from these three ratios show similar spatial variations, peaking at the centre of the nebula and decreasing from the centre to the outer regions. This object has the highest electron densities in our sample, with a mean density of $N_{\mathrm{e}}(\mathrm{S} \mathrm{II})=6811 \pm 613 \mathrm{~cm}^{-3}$, $N_{\mathrm{e}}(\mathrm{Cl}$ III $)=37694 \pm 5228 \mathrm{~cm}^{-3}$, and $N_{\mathrm{e}}($ Ar IV $)=10238 \pm$ $865 \mathrm{~cm}^{-3}$. In particular, the [Cl III] densities are higher than the $[\mathrm{S} \mathrm{II}]$ and $[\mathrm{Ar} I V]$ densities.

The [O III] and [N II] electron temperatures (see Fig. 15) also show well defined systematic variation along the nebular surface, with peak values of $T_{\mathrm{e}}(\mathrm{O}$ III $)=19865_{-54}^{+55} \mathrm{~K}$ and $T_{\mathrm{e}}(\mathrm{N}$ II $)=15407_{-127}^{+125} \mathrm{~K}$ in the central region of the nebula. The profiles of [O III] and [N II] electron temperatures are similar to each other, with the electron temperature increasing in the direction of the areas with higher surface brightness in $\mathrm{H} \beta$. Again, at the outskirts of the nebula higher temperatures are found. This nebula has also the highest electron temperatures in our sample, with mean values of $T_{\mathrm{e}}(\mathrm{O}$ III $)=18357 \pm 178 \mathrm{~K}$ and $T_{\mathrm{e}}(\mathrm{N}$ II $)=13798 \pm 163 \mathrm{~K}$.

\subsection{NGC 6563}

NGC 6563 is an object that appears on $\mathrm{H} \alpha$ images (Chu et al. 1987) as a fairly elliptical planetary nebula with brightening at the ends of the minor axis. Our observations have revealed that the electron temperature and density are relatively homogeneous (see Figs. 16 and 17) with mean values of 
$T_{\mathrm{e}}\left(\mathrm{N}_{\text {II }}\right)=10743 \pm 72 \mathrm{~K}$ and $N_{\mathrm{e}}\left(\mathrm{S}_{\mathrm{II}}\right)=134 \pm 10 \mathrm{~cm}^{-3}$. The low densities found here are evidence of a relatively evolved object. At the distance of $1867 \mathrm{kpc}$ (Maciel \& Pottasch 1980) and with an angular radius of 22.6" (Perek \& Kohoutek 1967), the expansion velocity of $11 \mathrm{~km} \mathrm{~s}^{-1}$ measured by Weinberger (1989) yields a dynamical age of $17.8 \times 10^{3} \mathrm{yr}$ for NGC 6563 .

\subsection{NGC 6781}

This is a planetary nebula with low surface brightness. Mavromatakis et al. (2001) mapped this nebula in several optical emission lines and these images display a known "C" like structure. The electron densities (see Fig. 18) are relatively low with a mean value of $N_{\mathrm{e}}(\mathrm{S}$ II $)=135 \pm 12 \mathrm{~cm}^{-3}$. Since the

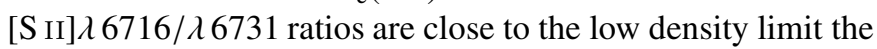
density measurements are more susceptible to errors; therefore the mean value derived of $N_{\mathrm{e}}(\mathrm{S}$ II $)=214 \mathrm{~cm}^{-3}$ by Liu et al. (2004) seems relatively compatible with our mean estimate. An age of $40000 \mathrm{yr}$ was determined by Mavromatakis et al. (2001) indicating that it is an evolved object. The electron temperatures distribution derived from the [N II] ratio is relatively uniform, with a mean of $T_{\mathrm{e}}(\mathrm{N}$ II $)=10763 \pm 140 \mathrm{~K}$, which is consistent with the findings by Mavromatakis et al. (2001) and Liu et al. (2004).

\subsection{NGC 6853}

The nebula NGC 6853, also known as Dumbbell Nebula, has a wide range of ionization as showed by Hawley \& Miller (1978). It is the nebula with the largest angular size in our sample. This object has a low surface brightness and irregular shape. NGC 6853 presents a relatively homogeneous electron density distribution (see Fig. 20), with a mean density of $N_{\mathrm{e}}(\mathrm{S}$ II) $=$ $95 \pm 7 \mathrm{~cm}^{-3}$. This is the object with the lowest density in our sample. The low densities in this nebula indicate that it is a relatively evolved object. The distribution of electron temperature (see Fig. 21) is about constant along the nebular surface. For the entire set of 142 apertures observed, we found a weighted mean electron temperature of $T_{\mathrm{e}}(\mathrm{N}$ II $)=10898 \pm 48 \mathrm{~K}$. Barker (1984) has measured the electron density and temperature in seven different positions for NGC 6853 finding a mean temperature of $T_{\mathrm{e}}\left(\mathrm{N}_{\text {II }}\right)=10529 \mathrm{~K}$, in accordance with our measurements.

\subsection{NGC 7009}

NGC 7009, sometimes called the Saturn Nebula, is a high surface brightness planetary nebula which has been well studied both observationally and theoretically. This nebula shows an elliptical structure with striking ansae (Aller 1941), that is, pairs of low ionization knots. Figure 22 shows the spatial variation of the electron density derived from the [S II], $[\mathrm{Cl} I I I]$, and $[\mathrm{Ar} \mathrm{IV}]$ ratios. The electron density derived from these three ratios presents a well defined variation of the density with the position along the nebular surface, with the density increasing in the direction of the higher surface brightness. We have estimated weighted mean electron densities of
$N_{\mathrm{e}}(\mathrm{S}$ II $)=3164 \pm 284 \mathrm{~cm}^{-3}, N_{\mathrm{e}}(\mathrm{Cl}$ III $)=6239 \pm 369 \mathrm{~cm}^{-3}$, and $N_{\mathrm{e}}(\operatorname{Ar} \mathrm{IV})=4314 \pm 872 \mathrm{~cm}^{-3}$.

The electron temperature estimates obtained from the [N II] and [O III] ratios and Balmer discontinuity (see Fig. 23) show systematic variation along the direction observed, with the temperature increasing with the increase of the surface brightness. We have derived mean temperatures of $T_{\mathrm{e}}\left(\mathrm{N}_{\text {II }}\right)=11163 \pm$ $196 \mathrm{~K}, T_{\mathrm{e}}(\mathrm{O}$ III $)=10117 \pm 146 \mathrm{~K}$, and $T_{\mathrm{e}}(\mathrm{Bal})=8589 \pm 109 \mathrm{~K}$. If we neglect the recombination excitation contribution to the $[\mathrm{N}$ II] $\lambda 5755$ line, the [N II] temperatures would be overestimated, particularly in the central parts of NGC 7009, and consequently we would obtain a spurious spatial gradient of [N II] temperature. Neglecting the correction for the recombination contribution to the $\left[\mathrm{N}_{\mathrm{II}}\right] \lambda 5755$ line, we would derive a mean temperature of $T_{\mathrm{e}}(\mathrm{N}$ II $)=13681 \pm 732 \mathrm{~K}$, which is about $2500 \mathrm{~K}$ higher than corrected value.

Many papers have been published about the physical properties of NGC 7009 (Gonçalves et al. 2003; Rubin et al. 2002; Liu et al. 1995; Barker 1983; Czyzak \& Aller 1979), and in general our estimates are compatible with previous determinations of temperatures and densities. Rubin et al. (2002) have evaluated the $[\mathrm{O} I I I]$ electron temperature variation along the major axis of NGC 7009, with spectrophotometric data obtained with the Hubble Space Telescope at a spatial resolution of 0.05 arcsec. Interestingly, they have also found a similar behaviour of temperature across the nebula. Gonçalves et al. (2003) have also presented estimates of temperature and density for eight different regions, along the major axis of NGC 7009. They have found the [O III] and [N II] electron temperatures remarkably constant throughout the nebula, with a average value of $T_{\mathrm{e}}(\mathrm{O}$ III $)=10200 \mathrm{~K}$ and $T_{\mathrm{e}}\left(\mathrm{N}_{\text {II }}\right)=$ $11100 \mathrm{~K}$.

\section{Discussion}

\subsection{Comparison of electron densities}

For five planetary nebulae in our sample, namely NGC 2440, NGC 3132, NGC 3242, NGC 6302, and NGC 7009, it was possible to compare the electron densities derived from the $[\mathrm{S} \mathrm{II}](\lambda 6716 / \lambda 6731),[\mathrm{Cl} \mathrm{III}](\lambda 5517 / \lambda 5537)$, and $[\operatorname{Ar} \operatorname{IV}](\lambda 4711 / \lambda 4740)$ ratio lines. It is important to realize that the $[\mathrm{SII}]$ ratio is a density sensor for the outer parts of the nebula, while the [ClIII] and [Ar IV] ratios are density indicators for the intermediate and inner parts of the nebula, respectively. From our estimates we have found that in general $N_{\mathrm{e}}(\mathrm{Cl}$ III $)>N_{\mathrm{e}}(\mathrm{Ar}$ IV $)>N_{\mathrm{e}}(\mathrm{S}$ II $)$ for NGC 2440 , NGC 6302 , and NGC 7009. As discussed by Stanghellini \& Kaler (1989), $N_{\mathrm{e}}(\mathrm{Cl}$ III $)>N_{\mathrm{e}}(\mathrm{Ar}$ IV) indicates that the [Ar IV] lines are produced in a rarefied inner region. For NGC 3132 and NGC 3242, the mean densities derived from the [ClIII] and [ArIV] ratios are approximately similar to each other; at the same time the densities determined from the [S II] ratio are substantially lower. These differences can occur because the $\mathrm{S}^{+}$zone is basically separated of both $\mathrm{Ar}^{+3}$ and $\mathrm{Cl}^{+2}$ zones. 


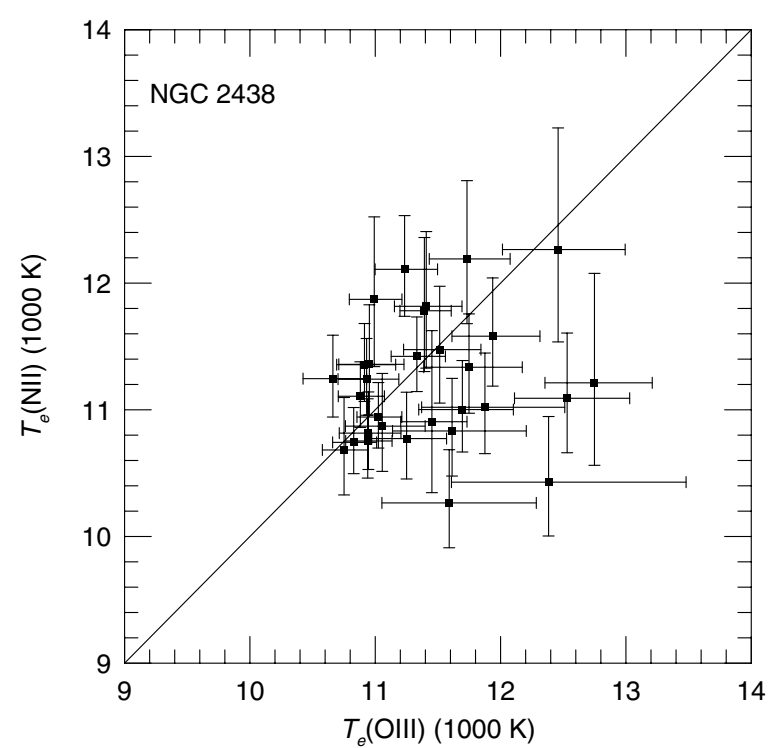

Fig. 24. $T_{\mathrm{e}}\left(\mathrm{N}\right.$ II) vs. $T_{\mathrm{e}}(\mathrm{O}$ III $)$ for NGC 2438.

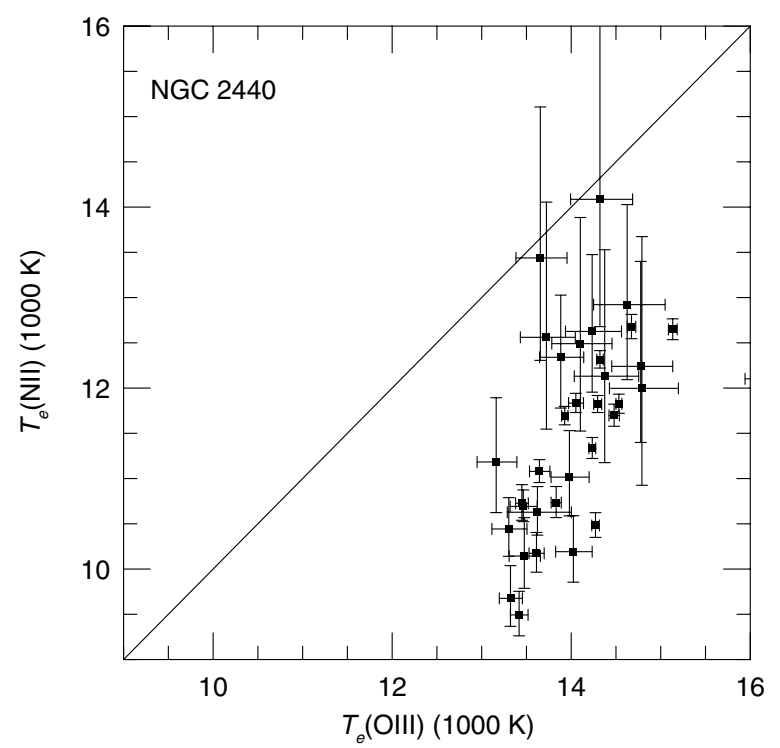

Fig. 25. Same as Fig. 24, but for NGC 2440.

\subsection{Comparison of electron temperatures}

Electron temperatures derived from different indicators can present considerable differences. These differences can be caused by temperature variations inside a given nebula. Kaler (1986) has showed that the mean ratio of $T_{\mathrm{e}}(\mathrm{N}$ II $) / T_{\mathrm{e}}(\mathrm{O}$ III $)$ varies as a function of overall nebular excitation. He found that as central star temperature increases from $T_{*} \sim 25000 \mathrm{~K}$ to $\sim 50000 \mathrm{~K}$, this ratio increases from $\sim 0.7$ to $\sim 1.1$, and then decreases to 0.7 again for the highest levels of excitation. In Figs. 24-28 we have plotted $T_{\mathrm{e}}\left(\mathrm{N}\right.$ II) versus $T_{\mathrm{e}}$ (O III) for NGC 2438 , NGC 2440, NGC 3132, NGC 6302, and NGC 7009.

We have found that $T_{\mathrm{e}}(\mathrm{N}$ II $)<T_{\mathrm{e}}(\mathrm{O}$ III) in NGC 2440 and NGC 6302, which are high excitation objects. For NGC 2438 and NGC 3132, which are low excitation objects, we verified that $T_{\mathrm{e}}(\mathrm{N}$ II $) \approx T_{\mathrm{e}}(\mathrm{O}$ III $)$. In the case of NGC $7009, T_{\mathrm{e}}\left(\mathrm{N}_{\mathrm{II}}\right)>$ $T_{\mathrm{e}}(\mathrm{O}$ III), with exception of two points.

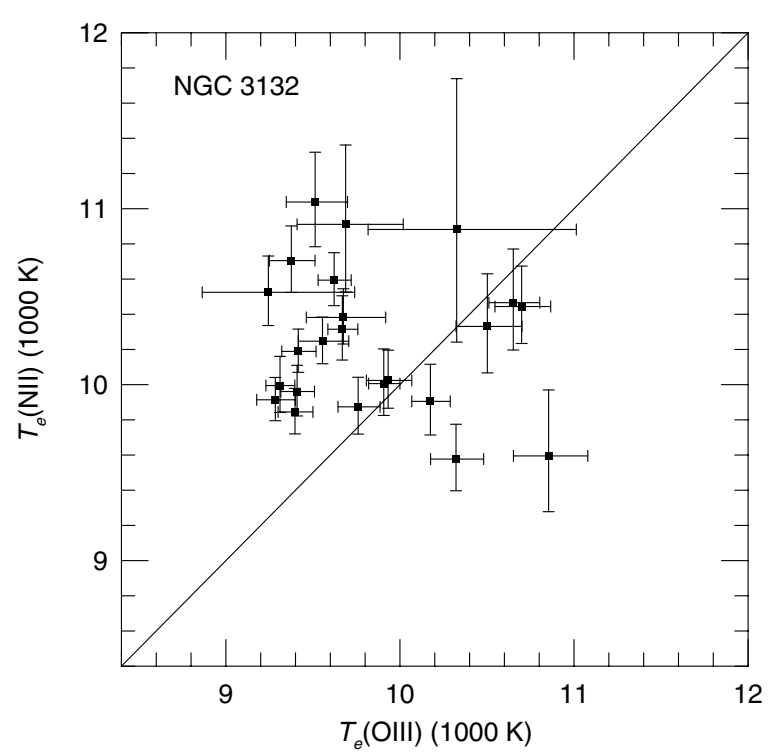

Fig. 26. Same as Fig. 24, but for NGC 3132.

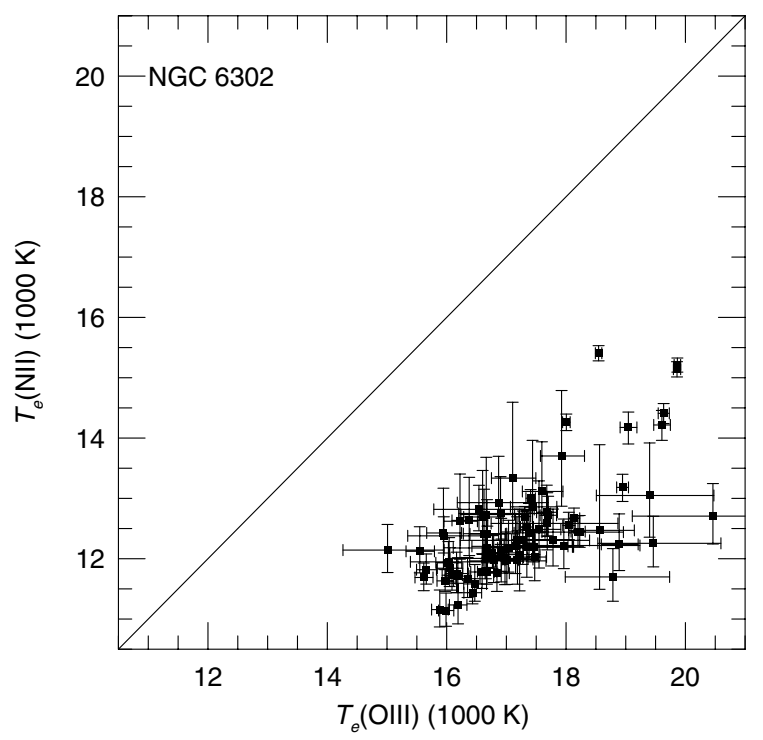

Fig. 27. Same as Fig. 24, but for NGC 6302.

Figures 5, 9, 13 and 23 show that $T_{\mathrm{e}}(\mathrm{O}$ III $)$ is systematically higher than $T_{\mathrm{e}}(\mathrm{Bal})$, although there are a few exceptions. On average, $T_{\mathrm{e}}(\mathrm{O}$ III $)$ is about $20 \%$ higher than $T_{\mathrm{e}}(\mathrm{Bal})$. As the nebular continuum emission is due to a recombination process, the $T_{\mathrm{e}}(\mathrm{Bal})$ values are weighted towards lower temperature regions, whereas the fluxes of collisionally excited lines are preferentially weighted towards higher temperature regions, so these higher [O III] temperatures are expected.

\subsection{Electron temperature versus density}

We found two different kinds of relationship between electron temperature and surface brightness or electron density. For NGC 2440, NGC 6302, and NGC 7009 we have found a correlation between electron temperature and electron density or surface brightness, with the electron temperature increasing with the increase of the electron density or surface brightness. 


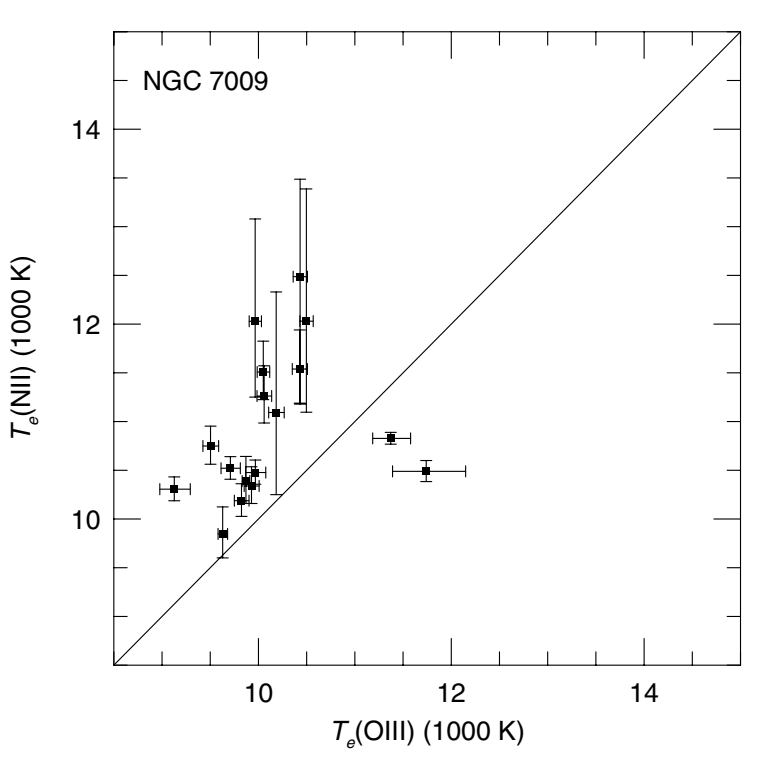

Fig. 28. Same as Fig. 24, but for NGC 7009.

For NGC 3242 and NGC 1535 we have also found a correlation between [O III] electron temperature and [Ar IV] electron density, although this correspondence is rather subtle. On the other hand, we have found an opposite relation between electron temperature and electron density for NGC 2438 and NGC 3132, with the $[\mathrm{O} I I I]$ electron temperature increasing with the decrease of the [S II] electron density, although this fact is not so clear for NGC 2438 because the [S II] ratios are close to the low density limit and this behaviour is somewhat masked. For the remaining objects NGC 6563, NGC 6781, and NGC 6853 it was not possible to detect any relationship between electron temperature and surface brightness. These objects present irregular surface brightness and the electron temperature and density profiles are fairly homogeneous. Figure 29 plots the $T_{\mathrm{e}}\left(\mathrm{O}\right.$ III) versus $N_{\mathrm{e}}(\mathrm{S}$ II) for NGC 2440 and NGC 6302.

The correlation and anti-correlation between temperature and density found in our sample of objects seem to be associated with the structure of the nebula. NGC 2438 and NGC 3132 , in which we have found the anti-correlation between temperature and density, are classical example of ring shaped nebulae. So, as a cavity is present in the central region of these nebulae higher densities are expected in the bright ring and lower densities in the central region of the nebula. An anticorrelation between [O III] temperature and density has also been found for the planetary nebula NGC 6720 (Garnett \& Dinerstein 2001; Guerrero et al. 1997), which is also a ring shaped nebula. On the other hand, NGC 2440, NGC 3242, NGC 6302, and NGC 7009, in which a correlation between temperature and density is present, are bipolar planetary nebulae that are denser in the central regions.

In most of objects (NGC 1535, NGC 2438, NGC 2440, NGC 3132, NGC 3242, and NGC 6302) we have found high temperatures at the outskirts of the nebula, where low surface brightnesses and densities are present. All these facts indicate that the density structure may play an important role in the production of temperature fluctuations.

\subsection{Magnitude of the electron temperature fluctuations}

In order to characterize temperature fluctuations and their effects on forbidden line abundance determination, Peimbert (1967) introduced the concepts of the mean ionic temperature $T_{0}$ and the temperature fluctuation parameter $t^{2}$, defined as

$t^{2}=\frac{\int\left(T_{\mathrm{e}}-T_{0}\right)^{2} N_{\mathrm{i}} N_{\mathrm{e}} \mathrm{d} V}{T_{0}^{2} \int N_{\mathrm{i}} N_{\mathrm{e}} \mathrm{d} V}$

$T_{0}=\frac{\int T_{0} N_{\mathrm{i}} N_{\mathrm{e}} \mathrm{d} V}{\int N_{\mathrm{i}} N_{\mathrm{e}} \mathrm{d} V}$,

where $N_{\mathrm{i}}$ is the density of the ion used to measure the temperature and the integrations are calculated over the observed volume of the nebula.

The $t^{2}$ parameter cannot be measured directly, so estimations have been obtained by comparing the abundances derived from forbidden and recombination lines. The values of $t^{2}$ required to reconcile the differences in the measurements of abundances determined by these two methods are high and lie in a range of $0.02 \leq t^{2} \leq 0.10$. For example, for the Orion Nebula, Esteban et al. (1999) estimated $t^{2} \approx 0.024$, while for NGC 7009, Liu et al. (1995) found $t^{2} \approx 0.1$.

A discrete estimation of $t^{2}$ can be obtained through pointto-point determinations of the electron temperature across the nebula, using the expression given by Liu (1998)

$t_{\mathrm{s}}^{2}=\frac{\sum_{i}\left(T_{\mathrm{e}}^{i}-T_{0}\right)^{2} F_{i}(\mathrm{H} \beta)}{T_{0}^{2} \sum_{i} F_{i}(\mathrm{H} \beta)}$

where $T_{\mathrm{e}}^{i}$ and $F_{i}(\mathrm{H} \beta)$ are the electron temperature and the $\mathrm{H} \beta$ flux obtained for the aperture $i$ respectively. Part of the observed relative variance $t_{\mathrm{s}}^{2}(\mathrm{obs})$ is due to errors in the measurements, so the final estimation of $t_{\mathrm{s}}^{2}$ must be corrected by $t_{\mathrm{s}}^{2}=t_{\mathrm{s}}^{2}(\mathrm{obs})-t_{\mathrm{er}}^{2}$, being $t_{\mathrm{er}}^{2}$ the relative mean quadratic error of the electron temperature measurements.

The measurements of temperature reported here correspond to mean values inside the apertures and along the line of sights, so any small-scale temperature fluctuation would be smoothed out by the present observations. For this reason, it is clear that $t_{\mathrm{s}}^{2}$ can only give a lower limit to $t^{2}$. However, the determination of $t_{\mathrm{s}}^{2}$ appears to be the only direct way to estimate $t^{2}$.

In Table 5 the values of $t_{\mathrm{s}}^{2}$ for the distributions of temperatures $T_{\mathrm{e}}(\mathrm{OIII}), T_{\mathrm{e}}(\mathrm{N}$ II $)$, and $T_{\mathrm{e}}(\mathrm{Bal})$ are presented. We have estimated values of $t_{\mathrm{s}}^{2}(\mathrm{Bal})$ ranging from 0.0003 to 0.0078 , $t_{\mathrm{s}}^{2}(\mathrm{~N}$ II $)$ from 0.0003 to 0.0097 , and $t_{\mathrm{s}}^{2}(\mathrm{O}$ III) from 0.0011 to 0.0050 .

Until now, low values of temperature fluctuations have also been found from point-to-point measurements of electron temperature for other objects: $t_{\mathrm{S}}^{2}(\mathrm{O} \mathrm{III})=0.002$ for the planetary nebula NGC 4361 (Liu 1998), $t_{\mathrm{s}}^{2}(\mathrm{O}$ III) $=0.0035$ for the planetary nebula NGC 7009 (Rubin et al. 2002), $t_{\mathrm{s}}^{2}(\mathrm{O}$ III) $=0.0025$ for the 30 Doradus Nebula (Krabbe \& Copetti 2002), and $t_{\mathrm{S}}^{2}(\mathrm{O}$ III $)=0.00682-0.0176$ and $t_{\mathrm{s}}^{2}(\mathrm{~N}$ II $)=0.00584-0.0175$ for Orion Nebula (Rubin et al. 2003). The low estimations of $t_{\mathrm{s}}^{2}$ found in this paper and also by other authors are also consistent 

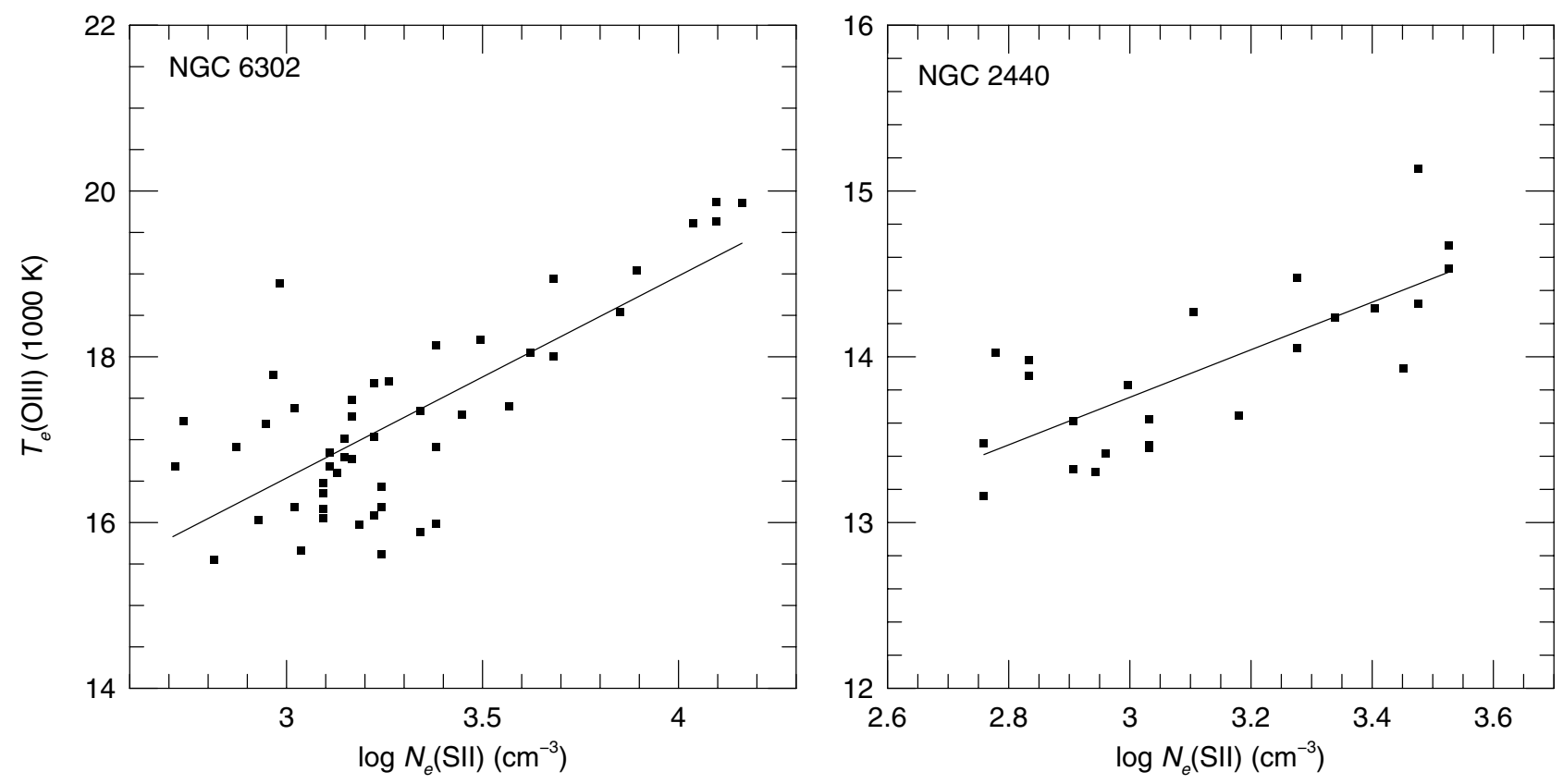

Fig. 29. $T_{\mathrm{e}}(\mathrm{O}$ III $)$ vs. $\log N_{\mathrm{e}}$ (S II) for NGC 2440 and NGC 6302. The data analyzed for these two objects are limited to the bright regions of the nebulae, where the correlation between temperature and density can be observed.

Table 5. Electron temperature fluctuations.

\begin{tabular}{lccc}
\hline \hline Object & $t_{\mathrm{s}}^{2}(\mathrm{Bal})$ & $t_{\mathrm{s}}^{2}(\mathrm{~N}$ II) & $t_{\mathrm{s}}^{2}(\mathrm{O}$ III) \\
\hline NGC 1535 & 0.0003 & & 0.0027 \\
NGC 2438 & & 0.0003 & 0.0032 \\
NGC 2440 & 0.0078 & 0.0041 & 0.0011 \\
NGC 3132 & & 0.0006 & 0.0047 \\
NGC 3242 & 0.0034 & & 0.0015 \\
NGC 6302 & & 0.0097 & 0.0050 \\
NGC 6563 & & 0.0005 & \\
NGC 6781 & & 0.0021 & \\
NGC 6853 & & 0.0016 & \\
NGC 7009 & 0.0008 & 0.0011 & 0.0023 \\
\hline
\end{tabular}

with those obtained from photoionization models (Kingdon 1995; Gruenwald \& Viegas 1995), but are too small to have a significant impact on the determination of abundance ratios derived from collisionally excited lines.

The difference found between mean [O III] and Balmer electron temperature also indicates considerable temperature fluctuations in NGC 1535, NGC 3242, and NGC 7009, and on the other hand, it indicates a low amplitude of temperature variations in NGC 2440. We have derived $t^{2}$ values from the expressions given by Peimbert (1967) relating [O III] and Balmer temperatures as a function of $T_{0}$ and $t^{2}$, assuming the same values for $T_{0}$ and $t^{2}$ in the $\mathrm{H}^{+}$and $\mathrm{O}^{++}$zones, and we have found $t^{2}=0.0523$ for NGC $1535, t^{2}=0.0085$ for NGC 2440, $t^{2}=0.0564$ for NGC 3242, and $t^{2}=0.050$ for NGC 7009 .

Some evidences against the presence of high temperature fluctuations were presented by Liu et al. (2000). They have derived abundances of metals in the planetary nebula NGC 6153 from infrared emission lines, which are almost independent of temperature. Since these abundances were very similar to those derived from ultraviolet and optical forbidden lines, Liu et al. (2000) have rejected the presence of large temperature fluctuations in NGC 6153 and pointed out the presence of hydrogen deficient clumps within the nebula, where the heavy element recombination lines would be strongly enhanced, as a possible cause of the discrepancies between abundances derived from recombination and forbidden lines. On other hand, Ruiz et al. (2003) and Peimbert et al. (2004) have supported the hypothesis of high temperature variations in gaseous nebulae and some evidences pointed out by them are: the large differences between the Balmer and [O III] temperatures found in gaseous nebulae (Peimbert 1971; Liu \& Danziger 1993; Zhang et al. 2004) and the differences of the $\mathrm{He}^{+} / \mathrm{H}^{+}$values derived from different helium lines under the assumption of a constant electron temperature (Peimbert et al. 2004; Peimbert et al. 1995), indicating the presence of high temperature variations. Other potential evidence in favor of high temperature fluctuations was presented by O'Dell et al. (2003). These authors measured the [O III] electron temperature in $1.5 \times 10^{6}$ different positions in the Orion Nebula based on data obtained with the Hubble Space Telescope and they found that $t_{\mathrm{s}}^{2}(\mathrm{O}$ III $)=0.008$. From this value and some geometrical considerations they estimated $t^{2}=0.028 \pm 0.006$.

Although we have found temperature fluctuations of low amplitude for the planetary nebulae of our sample, we cannot completely rule out the existence of larger temperature fluctuations. As our measurements of electron temperature are averages along the lines of sight, $t_{\mathrm{s}}^{2}$ is an inferior limit of $t^{2}$ and higher values of electron temperature fluctuations can possibly exist in these nebulae. 


\section{Conclusions}

An observational study on the variation of electron temperature and density has been conducted in 10 galactic planetary nebulae, using long slit spectrophotometry of high signalto-noise ratio in the range of 3100 to $6900 \AA$. Electron temperatures were determined from the [O III $](\lambda 4959+$ $\lambda 5007) / \lambda 4363$ and $[\mathrm{N} \mathrm{II}](\lambda 6548+\lambda 6583) / \lambda 5755$ ratios and the Balmer discontinuity and electron densities were estimated from the $[\mathrm{S}$ II] $\lambda 6716 / \lambda 6731,[\mathrm{Cl}$ III] $\lambda 5517 / \lambda 5537$, and $[\operatorname{ArIV}] \lambda 4711 / \lambda 4740$ ratios. The main findings are the following:

1. The electron temperature estimates obtained from $[\mathrm{O}$ III $](\lambda 4959+\lambda 5007) / \lambda 4363$ and $[\mathrm{N}$ II $](\lambda 6548+$ $\lambda 6583) / \lambda 5755$ ratios and observed Balmer discontinuity present systematic variation along the nebular surface in most of the objects studied (NGC 1535, NGC 2438, NGC 2440, NGC 3132, NGC 3242, NGC 6302, and NGC 7009). In most of the cases, the electron temperature decreases from the center to the edges, with higher temperatures also found at the outskirts of the nebulae. For NGC 6563, NGC 6781, and NGC 6853 a uniform distribution of electron temperature have been observed.

2. Electron temperature fluctuations of low amplitude have been observed in the planetary nebulae studied here. The temperature distribution across each nebula presents a variance relative to the mean corresponding to $0.0003 \leq$ $t_{\mathrm{s}}^{2}(\mathrm{Bal}) \leq 0.0078,0.0003 \leq t_{\mathrm{s}}^{2}(\mathrm{~N}$ II $) \leq 0.0097$, and $0.0011 \leq t_{\mathrm{s}}^{2}(\mathrm{O}$ III $) \leq 0.0050$. Although we have found temperature fluctuations of low amplitude for these nebulae, we cannot completely rule out the existence of larger temperature fluctuations. As our measurements of electron temperature are averages along the lines of sight, $t_{\mathrm{s}}^{2}$ is an inferior limit of $t^{2}$ and higher electron temperature fluctuations can possibly exist in these nebulae.

3. A systematic spatial variation of electron density has been detected in most of objects studied. For NGC 2438, NGC 3132 , and NGC 6563 , the density profile presents a double peaked distribution along the nebular surface. For NGC 1535, NGC 2440, NGC 3242, NGC 6302, and NGC 7009 the electron density is decreasing from the center to the edges. The remaining objects (NGC 6781 and NGC 6853) have not shown any significant electron density dependence on position. NGC 2438, NGC 6563, NGC 6781, and NGC 6853 are in general the most diffuse and probably evolved objects studied here, with low mean densities in the range $N_{\mathrm{e}}(\mathrm{S}$ II $) \approx 95-158 \mathrm{~cm}^{-3}$.

4. An anti-correlation between temperature and density was found for NGC 2438 and NGC 3132, with the electron temperature increasing with the decrease of electron density and a correlation between temperature and density was found for NGC 1535, NGC 2440, NGC 3242, NGC 6302, and NGC 7009, with the electron temperature increasing with the increase of electron density. These relationships seem to be associated with the morphology of the nebula. The nebulae in which the correlation between temperature and density is present are ring shaped. The anticorrelation between temperature and density is found in bipolar planetary nebulae that are denser in the centre of the nebula.

Acknowledgements. This paper is based on observations made with the $1.52 \mathrm{~m}$ and $1.60 \mathrm{~m}$ telescope, obtained at the ESO and LNA, respectively. We are grateful to the Brazilian institutions CAPES, CNPQ and LNA for the partially supporting this work.

\section{References}

Aller, L. H. 1941, ApJ, 93, 236

Aller, L. H., \& Walker, M. F. 1965, ApJ, 141, 1318

Aller, L. H., Ross, J. E., O’Mara, B. J., \& Keys, C. D. 1981, MNRAS, 197, 95

Baessgen, M., Diesch, C., \& Grewing, M. 1990, ApJ, 237, 201

Barker, T. 1983, ApJ, 267, 630

Barker, T. 1984, ApJ, 284, 589

Benjamin, B. A., Skillman, E. D., \& Smits, D. P. 1999, ApJ, 514, 307

Bernard Salas, J., Pottasch, S. R., Beintema, D. A., \& Wesselius, P. R. 2001, A\&A, 367, 949

Boeshaar, G. O. 1974, ApJ, 187, 283

Bowen, I. S. 1960, ApJ, 132, 1

Butler, K., \& Zeippen, C. J. 1989, A\&A, 208, 337

Cahn, J. H., \& Kaler, J. B. 1971, ApJS, 22, 319

Cahn, J. H., Kaler, J. B., \& Stanghellini, L. 1992, A\&AS, 94, 399

Chu, Y., Jacoby, G. H., \& Arendt, R. 1987, ApJS, 64, 529

Czyzak, S. J., \& Aller, L. H. 1979, MNRAS, 188, 229

Esteban, C., Peimbert, M., Torres-Peimbert, S., \& Rodríguez, M. 1999, Rev. Mex. Astron. Astrofis., 35, 65

Garnett, D. R, \& Dinerstein, H. L. 2001, ApJ, 558, 145

Giuliani, J. L. 1981, ApJ, 245, 90

Gonçalves, D. R., Corradi, R. L. M., Mampaso, A., \& Perinotto, M. 2003, ApJ, 597, 975

Guerrero, M. A., Manchado, A., \& Chu, Y.-H. 1997, ApJ, 487, 328

Guerrero, M. A., \& Manchado, A. 1999, ApJ, 522, 378

Gum, C. S. 1955, Mem. R. Astron. Soc., 67, 155

Gutierrez-Moreno, A., Cortes, G., \& Moreno, H. 1985, PASP, 97, 397

Gruenwald, R., \& Viegas, S. M. 1995, A\&A, 303, 535

Hawley, S. A., \& Miller, J. S. 1978, PASP, 90, 39

Hyung, S., \& Aller, L. H. 1998, PASP, 110, 466

Hummer, D. G., Storey, P. J. 1987, MNRAS, 224, 801

Juguet, J. L., Louise, R., Macron, A., \& Pascoli, G. 1988, A\&A, 205, 267

Kaler, J. B. 1986, ApJ, 308, 322

Kaufman, V., \& Sugar, J. 1986, JPCRD, 15, 321

Keenan, F. P., Hibbert, A., Ojha, P. C., \& Conlon, E. S. 1993, Phys. Scr. A, 48, 129

Kingdon, J. B., \& Ferland, G. J. 1995, ApJ, 450, 691

Krabbe, A. C., \& Copetti, M. V. F. 2002, A\&A, 387, 29

Lennon, D. J., \& Burke, V. M. 1994, A\&AS, 103, 273

Liu, X.-W. 1998, MNRAS, 295, 699

Liu, X.-W., \& Danziger, I. J. 1993, MNRAS, 263, 256

Liu, X.-W., Storey, P. J., Barlow, M. J., \& Clegg, R. E. S. 1995, MNRAS, 272, 369

Liu, X.-W., Storey, P. J., Danziger, I. J., Cohen, M., \& Bryce, M. 2000, MNRAS, 312, 585

Liu, X.-W., Luo, S.-G., Barlow, M. J., Danziger, I. J., \& Storey, P. J. 2001, MNRAS, 327, 141

Liu, Y., Liu, X.-W., Luo, S.-G., \& Barlow, M. J. 2004, MNRAS, 353, 1231

Maciel, W. J., \& Pottasch, S. R. 1980, A\&A, 881

Mavromatakis, F., Papamastorakis, J., \& Paleologou, E. V. 2001, A\&A, 374, 280 
Meatheringham, S. J., Wood, P. R., \& Faulkner, D. J. 1988, ApJ, 334, 862

Mendoza, C. 1983, Planetary Nebulae, IAU Symp., 103, 143

Mendoza, C., \& Zeippen, C. J. 1982, MNRAS, 198,127

Minkowski, R., \& Aller, L. H. 1956, ApJ, 124, 93

Moore, C. E. 1985, Selected Tables of Atomic Spectra, NSRDS-NBS 3 , Sect. 11

O'Dell, C. R., Peimbert, M., \& Peimbert, A. 2003, AJ, 125, 2590

Osterbrock, D. E. 1989, Astrophysics of Gaseous Nebulae and Active Galactic Nuclei, University Science Books, Mill Valley, CA

Peimbert, M. 1967, ApJ, 150, 825

Peimbert, M. 1971, Bol. Obs. Tonantzintla Tacubaya, 6, 29

Peimbert, M. 1978, Planetary Nebulae, IAU Symp., 76, 215

Peimbert, M., \& Torres-Peimbert, S. 1983, Planetary Nebulae, IAU Symp., 103, 233

Peimbert, M., \& Torres-Peimbert, S. 1987, Rev. Mex. Astron. Astrofis., 14, 540

Peimbert, M., Torres-Peimbert, S., \& Luridiana, V. 1995, Rev. Mex. Astron. Astrofis., 31, 131

Peimbert, M., Peimbert, A., Ruiz, M. T., \& Esteban, C. 2004, ApJS, 150,431

Perek, L., \& Kohoutek, L. 1967, Acad. Publ. House Czech. Acad. Sci., 1,1

Pottasch, S. R. 1983, Planetary Nebulae, IAU Symp., 103, 391

Ramsbottom, C. A., Bell, K. L., \& Stafford, R. P. 1996, ADNDT, 63, 57
Rola, C., \& Pelat, D. 1994, A\&A, 287, 676

Rowlands, N., Houck, J. R., Herter, T., Gull, G. E., \& Skrutskie, M. F. 1989, ApJ, 341, 901

Rubin, R. H., Bhatt, N. J., Dufour, R. J., et al. 2002, MNRAS, 334, 777

Rubin, R. H., Martin, P. G., Dufour, R. J., et al. 2003, MNRAS, 340, 362

Ruiz, M. T., Peimbert, A., Peimbert, M., \& Esteban, C. 2003, ApJ, 595,247

Savage, B. D., \& Mathis, J. S. 1979, ARA\&A, 17, 73

Shields, G. A., Aller, L. H., Keyes, C. D., \& Czyak, S. J. 1981, ApJ, 248, 569

Stanghellini, L., \& Kaler, J. B. 1989, ApJ, 343, 811

Torres-Peimbert, S., \& Peimbert, M. 1977, Rev. Mex. Astron. Astrofis., 2, 181

Verner D. A., Verner, E. M., \& Ferland, G. J. 1996, ADNDT, 64, 1

Zeippen, C. J., Le Bourlot, J., \& Butler, K. 1987, A\&A, 188, 251

Zhang, Y., Liu, X.-W., Wesson, R., et al. 2004, MNRAS, 351, 935

Walter, D. K., Dufour, R. J., \& Hester, J. J. 1992, ApJ, 397, 196

Weinberger, R. 1989, A\&AS, 78, 301

Wiese, W. L., Fuhr, J. R., \& Deters, T. M. 1996, JPCRD, Monograph, 7

Williams, R. E., \& Livio, M 1995, Proc. STScI, 8, 24 
A. C. Krabbe and M. V. F. Copetti: Electron temperature fluctuations, Online Material $p 1$

\section{Online Material}


A. C. Krabbe and M. V. F. Copetti: Electron temperature fluctuations, Online Material p 2

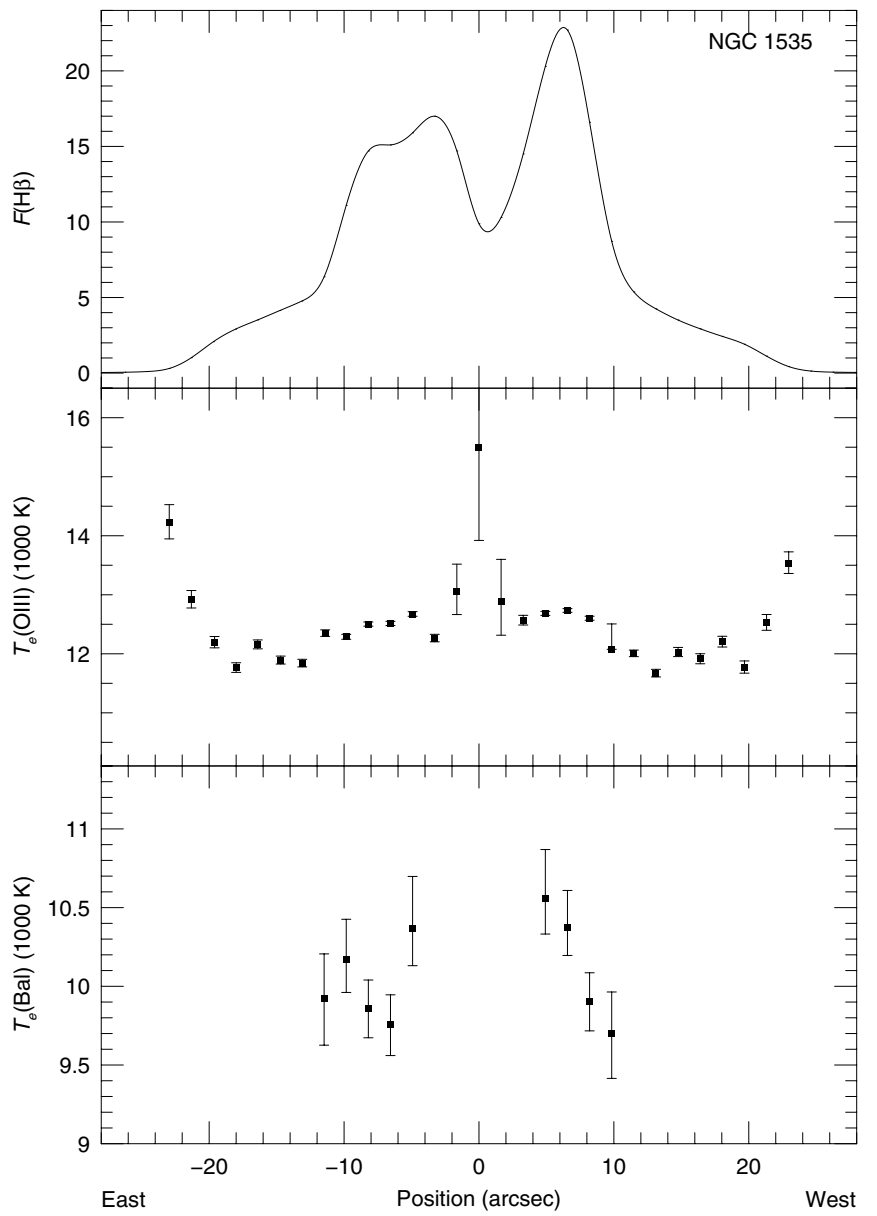

Fig. 5. NGC 1535. Spatial profiles of $\mathrm{H} \beta$ flux (in units of $\left.10^{-14} \mathrm{erg} \mathrm{cm}^{-2} \mathrm{~s}^{-1}\right), T_{\mathrm{e}}(\mathrm{O}$ III $)$ and $T_{\mathrm{e}}(\mathrm{Bal})$.

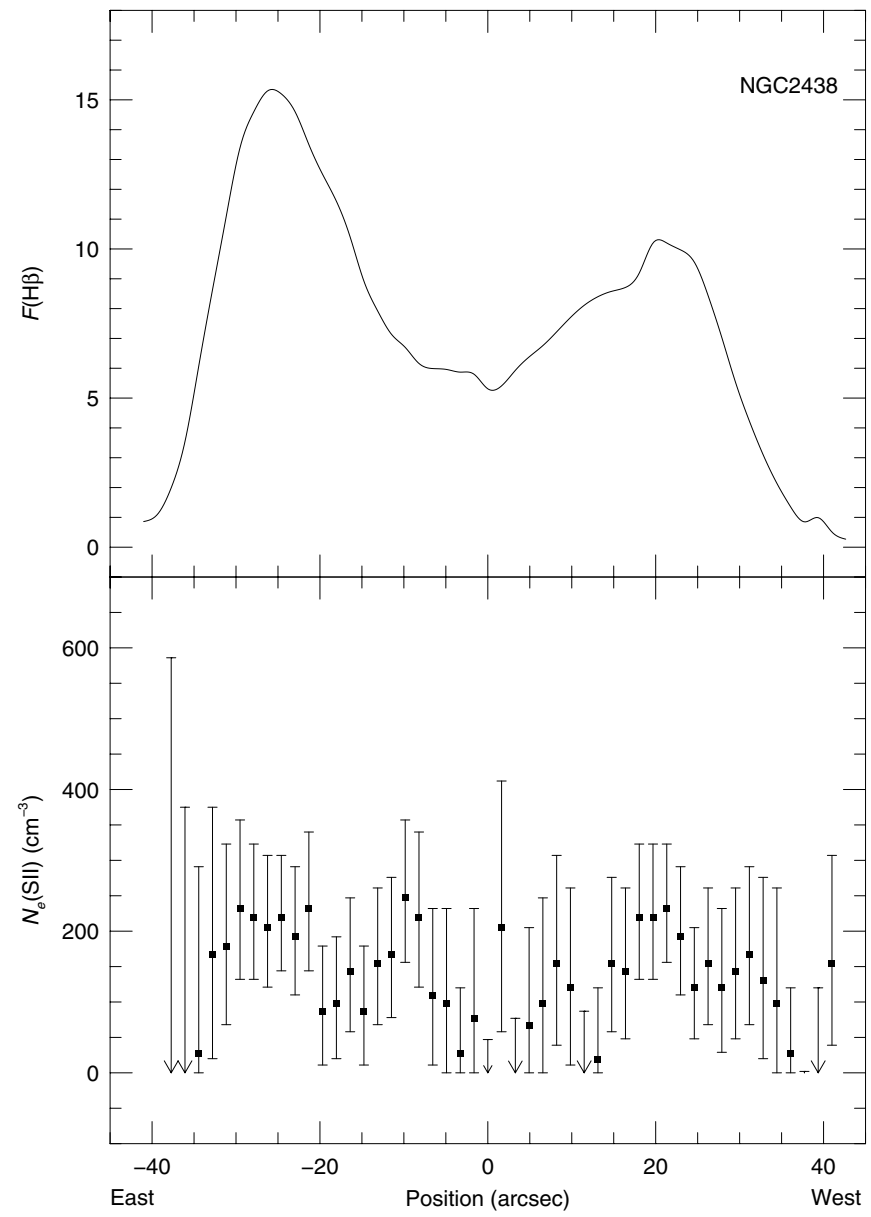

Fig. 6. NGC 2438. Spatial profiles of $\mathrm{H} \beta$ flux (in units of $\left.10^{-15} \mathrm{erg} \mathrm{cm}^{-2} \mathrm{~s}^{-1}\right)$ and $N_{\mathrm{e}}(\mathrm{S} \mathrm{II})$. 
A. C. Krabbe and M. V. F. Copetti: Electron temperature fluctuations, Online Material p 3

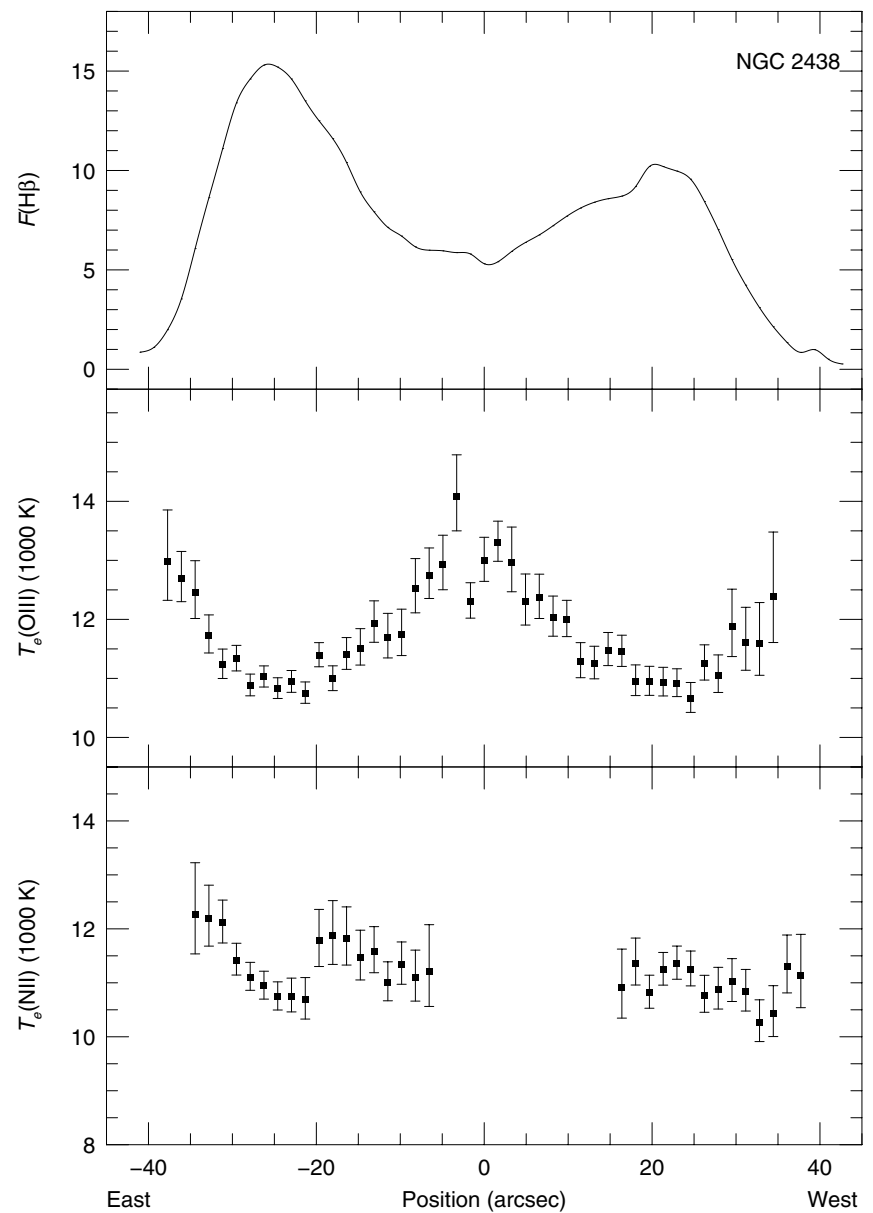

Fig. 7. NGC 2438. Spatial profiles of $\mathrm{H} \beta$ flux (in units of $\left.10^{-15} \mathrm{erg} \mathrm{cm}^{-2} \mathrm{~s}^{-1}\right), T_{\mathrm{e}}(\mathrm{O}$ III $)$ and $T_{\mathrm{e}}(\mathrm{N} \mathrm{II})$.

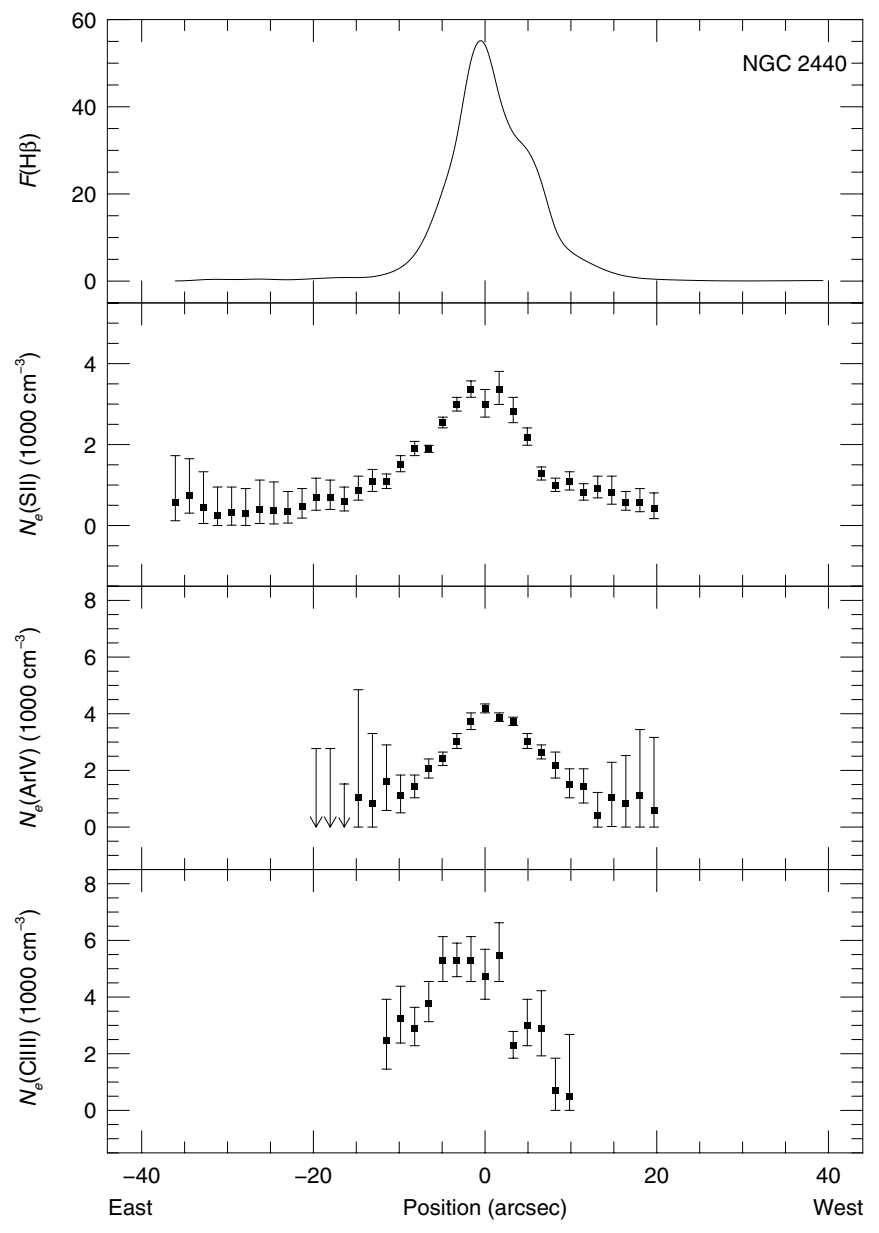

Fig. 8. NGC 2440. Spatial profiles of $\mathrm{H} \beta$ flux (in units of $\left.10^{-14} \mathrm{erg} \mathrm{cm}^{-2} \mathrm{~s}^{-1}\right), N_{\mathrm{e}}(\mathrm{S} \mathrm{II}), N_{\mathrm{e}}(\mathrm{Cl}$ III $)$ and $N_{\mathrm{e}}(\mathrm{Ar} \mathrm{IV})$. 
A. C. Krabbe and M. V. F. Copetti: Electron temperature fluctuations, Online Material p 4

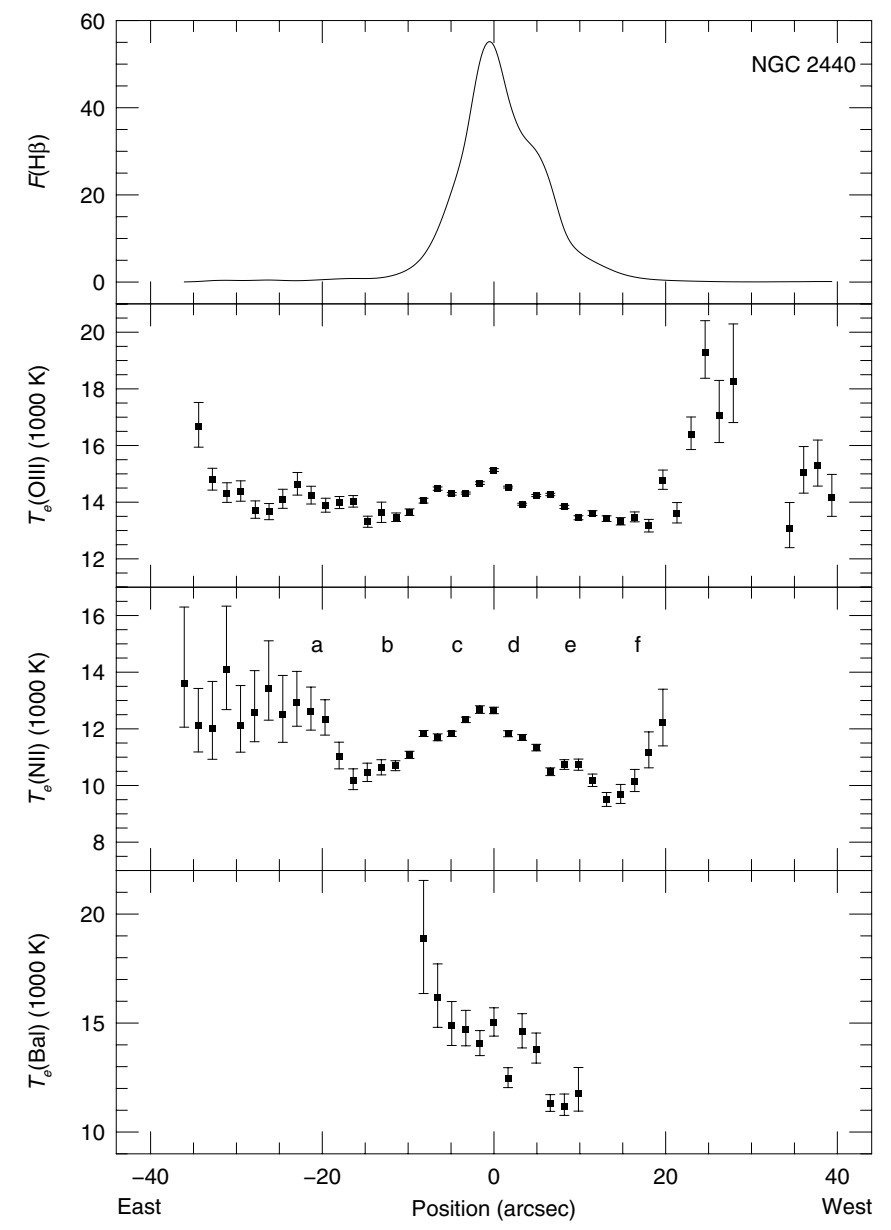

Fig. 9. NGC 2440. Spatial profiles of $\mathrm{H} \beta$ flux (in units of $\left.10^{-14} \mathrm{erg} \mathrm{cm}^{-2} \mathrm{~s}^{-1}\right), T_{\mathrm{e}}(\mathrm{O} \mathrm{III}), T_{\mathrm{e}}(\mathrm{N}$ II $)$ and $T_{\mathrm{e}}(\mathrm{Bal})$.

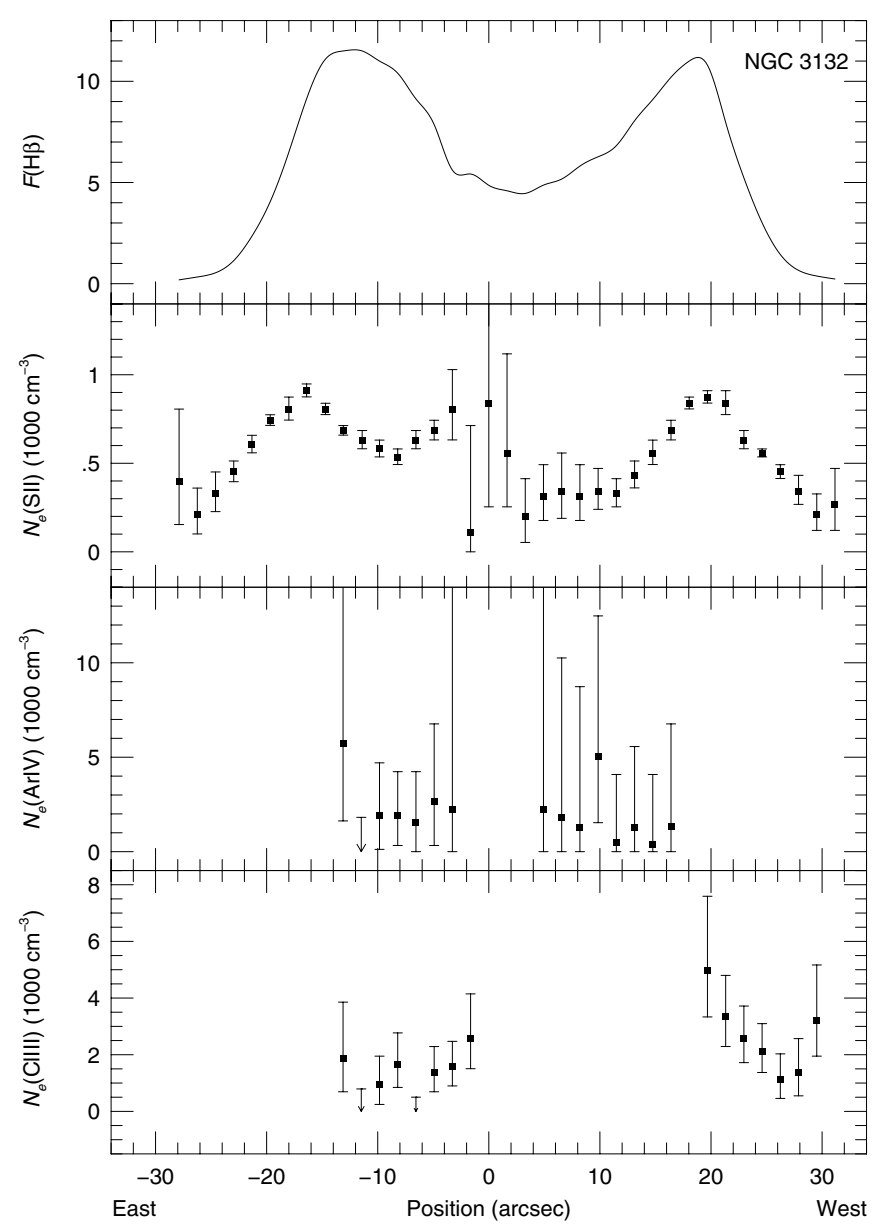

Fig. 10. NGC 3132. Spatial profiles of $\mathrm{H} \beta$ flux (in units of $\left.10^{-14} \mathrm{erg} \mathrm{cm}^{-2} \mathrm{~s}^{-1}\right), N_{\mathrm{e}}(\mathrm{S} \mathrm{II}), N_{\mathrm{e}}(\mathrm{Cl} \mathrm{III})$ and $N_{\mathrm{e}}(\mathrm{Ar} \mathrm{IV})$. 
A. C. Krabbe and M. V. F. Copetti: Electron temperature fluctuations, Online Material p 5

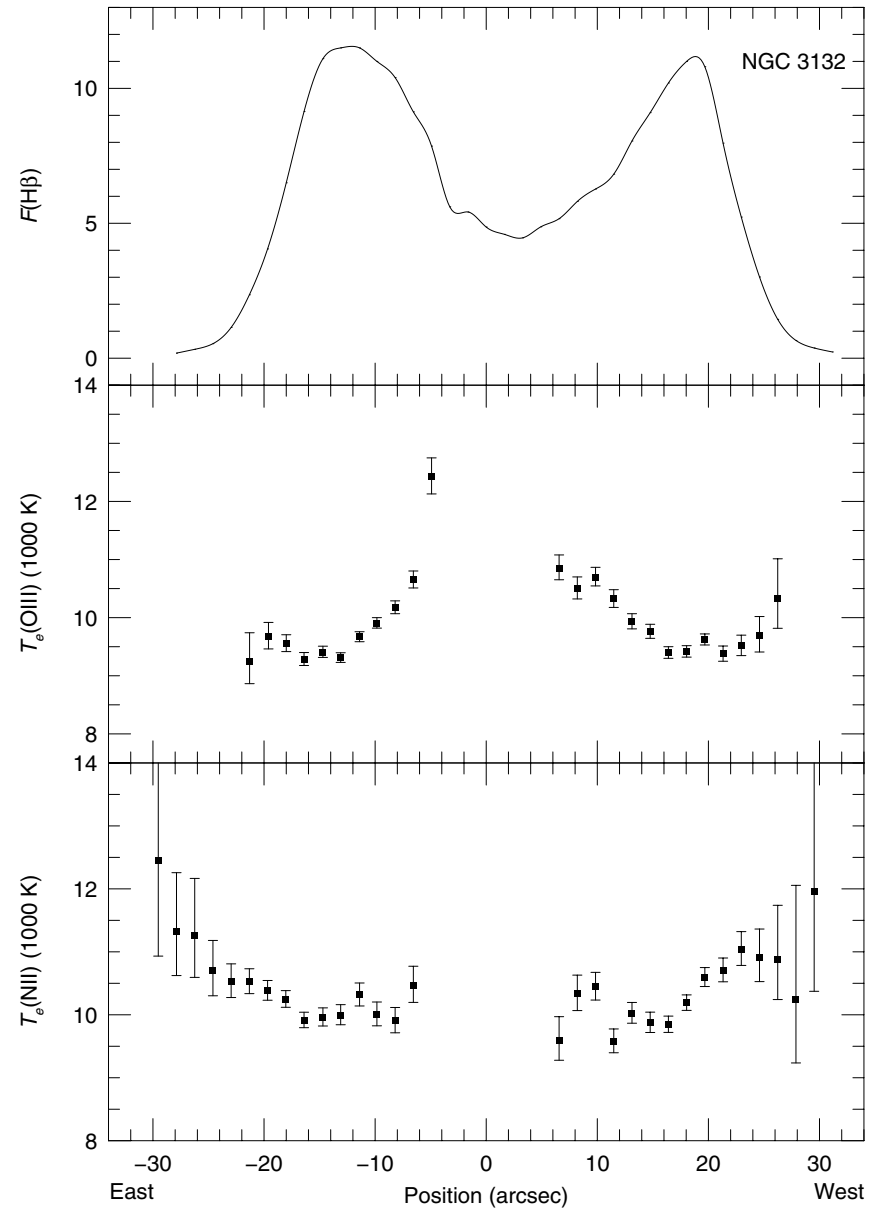

Fig. 11. NGC 3132. Spatial profiles of $\mathrm{H} \beta$ flux (in units of $10^{-14} \mathrm{erg}$ $\left.\mathrm{cm}^{-2} \mathrm{~s}^{-1}\right), T_{\mathrm{e}}(\mathrm{O}$ III $)$ and $T_{\mathrm{e}}(\mathrm{N}$ II $)$.

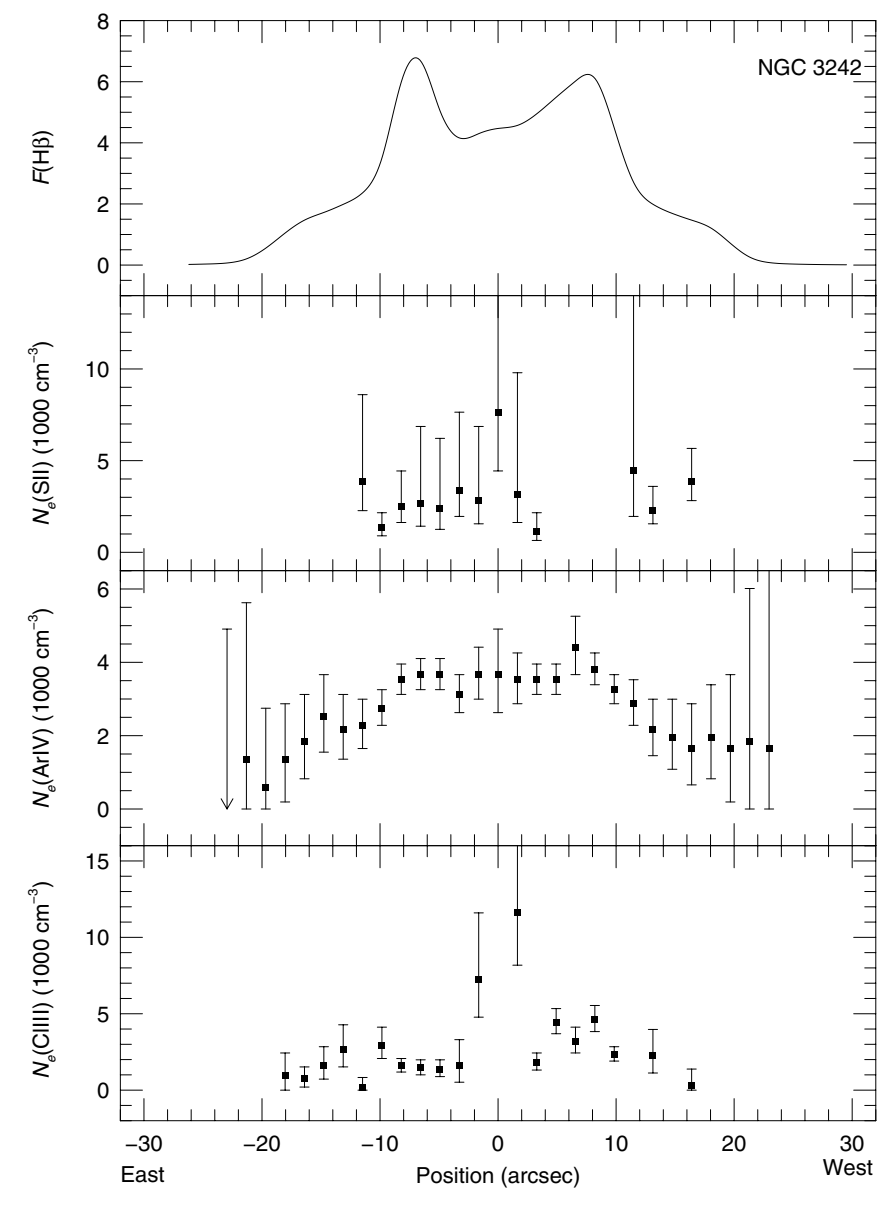

Fig. 12. NGC 3242. Spatial profiles of $\mathrm{H} \beta$ flux (in units of $10^{-13} \mathrm{erg}$ $\left.\mathrm{cm}^{-2} \mathrm{~s}^{-1}\right), N_{\mathrm{e}}(\mathrm{S} \mathrm{II}), N_{\mathrm{e}}(\mathrm{Cl} \mathrm{III})$ and $N_{\mathrm{e}}(\mathrm{Ar} \mathrm{IV})$. 
A. C. Krabbe and M. V. F. Copetti: Electron temperature fluctuations, Online Material p 6

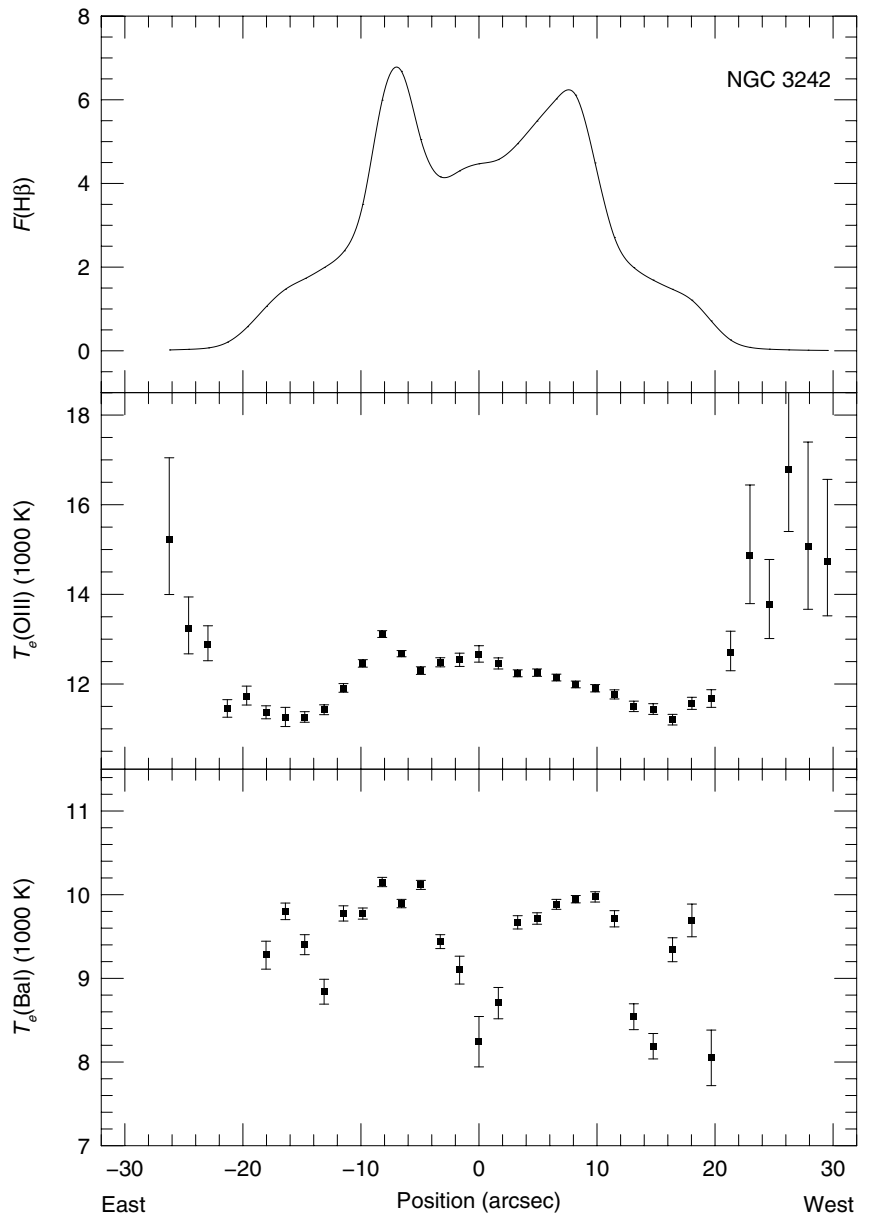

Fig. 13. NGC 3242. Spatial profiles of $\mathrm{H} \beta$ flux (in units of $10^{-13} \mathrm{erg}$ $\left.\mathrm{cm}^{-2} \mathrm{~s}^{-1}\right), T_{\mathrm{e}}(\mathrm{O}$ III $)$ and $T_{\mathrm{e}}(\mathrm{Bal})$.

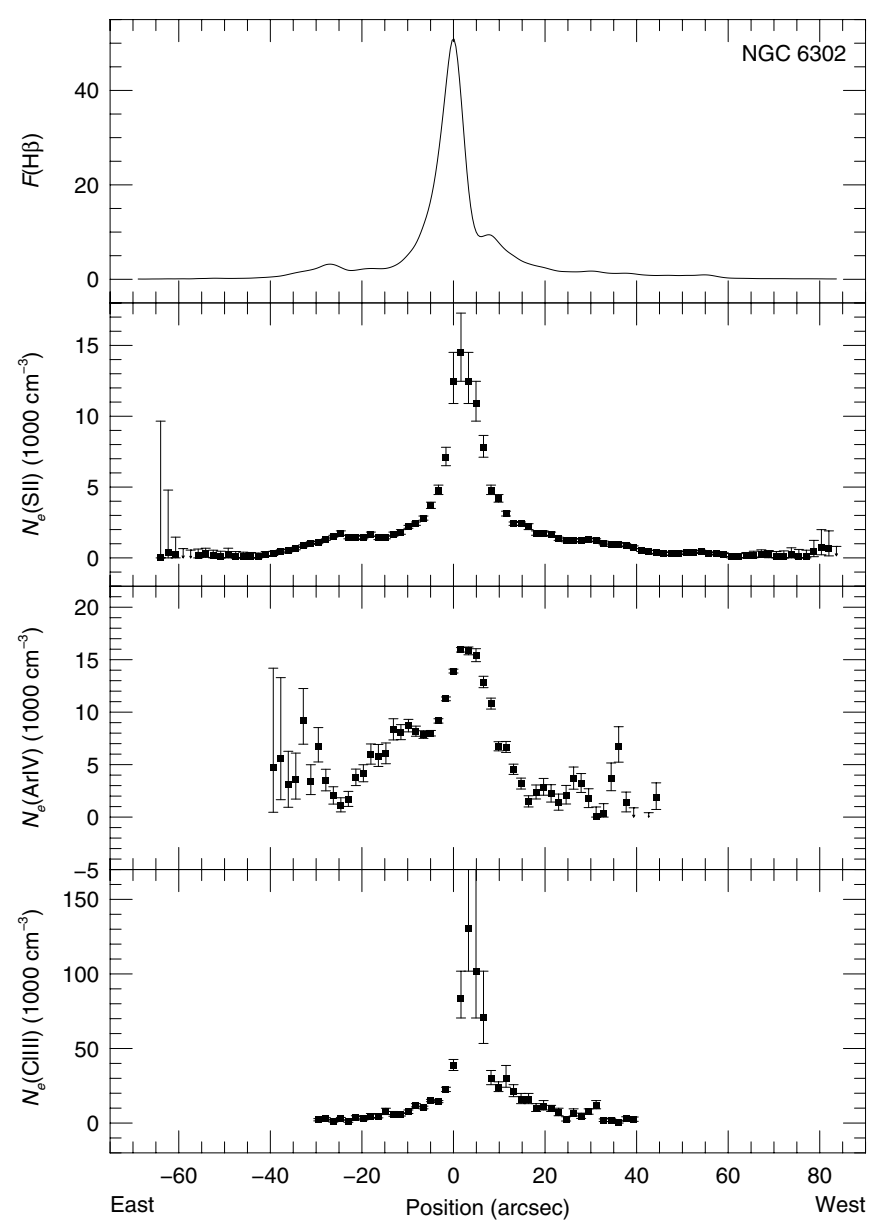

Fig. 14. NGC 6302. Spatial profiles of $\mathrm{H} \beta$ flux (in units of $10^{-14} \mathrm{erg}$ $\left.\mathrm{cm}^{-2} \mathrm{~s}^{-1}\right), N_{\mathrm{e}}(\mathrm{S}$ II $), N_{\mathrm{e}}(\mathrm{Ar} \mathrm{IV})$ and $N_{\mathrm{e}}(\mathrm{Cl} \mathrm{III})$. 
A. C. Krabbe and M. V. F. Copetti: Electron temperature fluctuations, Online Material p 7

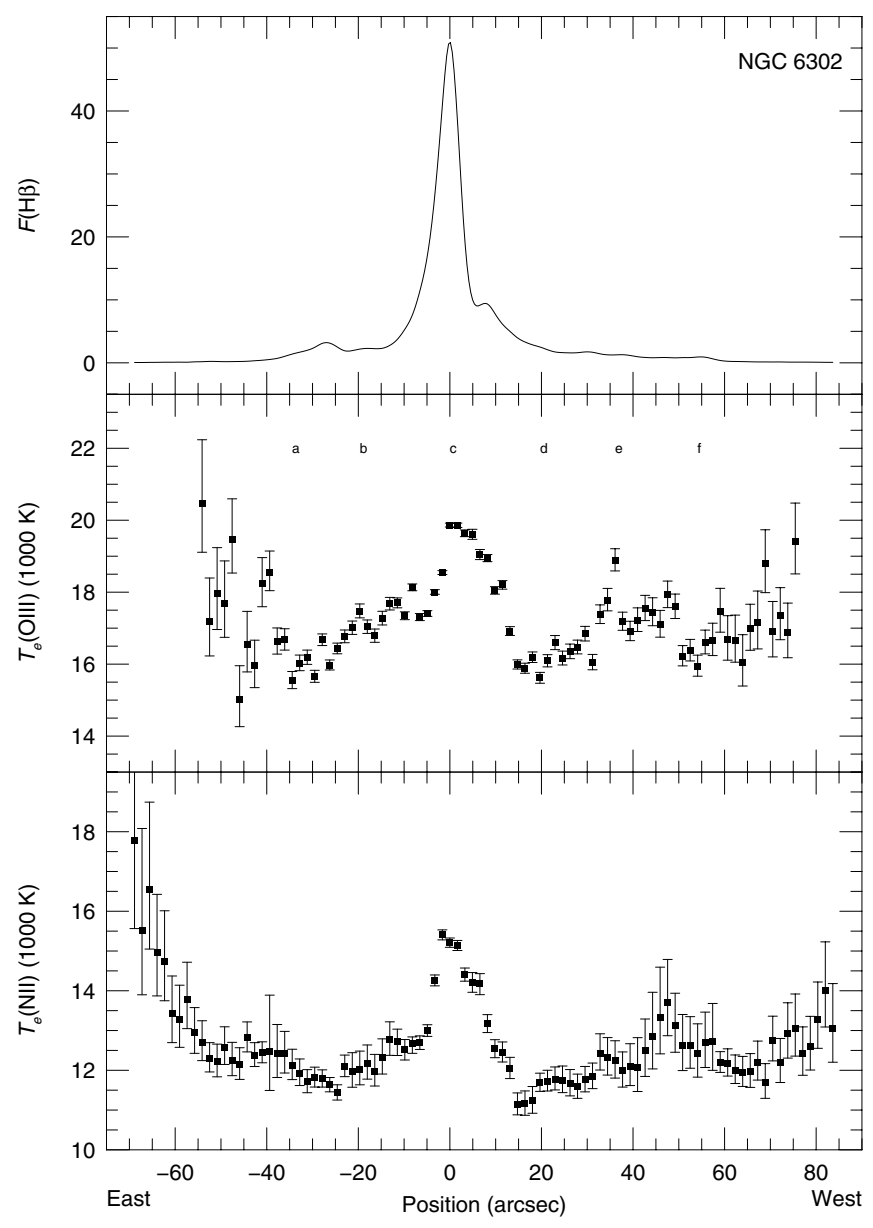

Fig. 15. NGC 6302. Spatial profiles of $\mathrm{H} \beta$ flux (in units of $10^{-14} \mathrm{erg}$ $\left.\mathrm{cm}^{-2} \mathrm{~s}^{-1}\right), T_{\mathrm{e}}(\mathrm{O}$ III $)$ and $T_{\mathrm{e}}(\mathrm{N}$ II $)$.

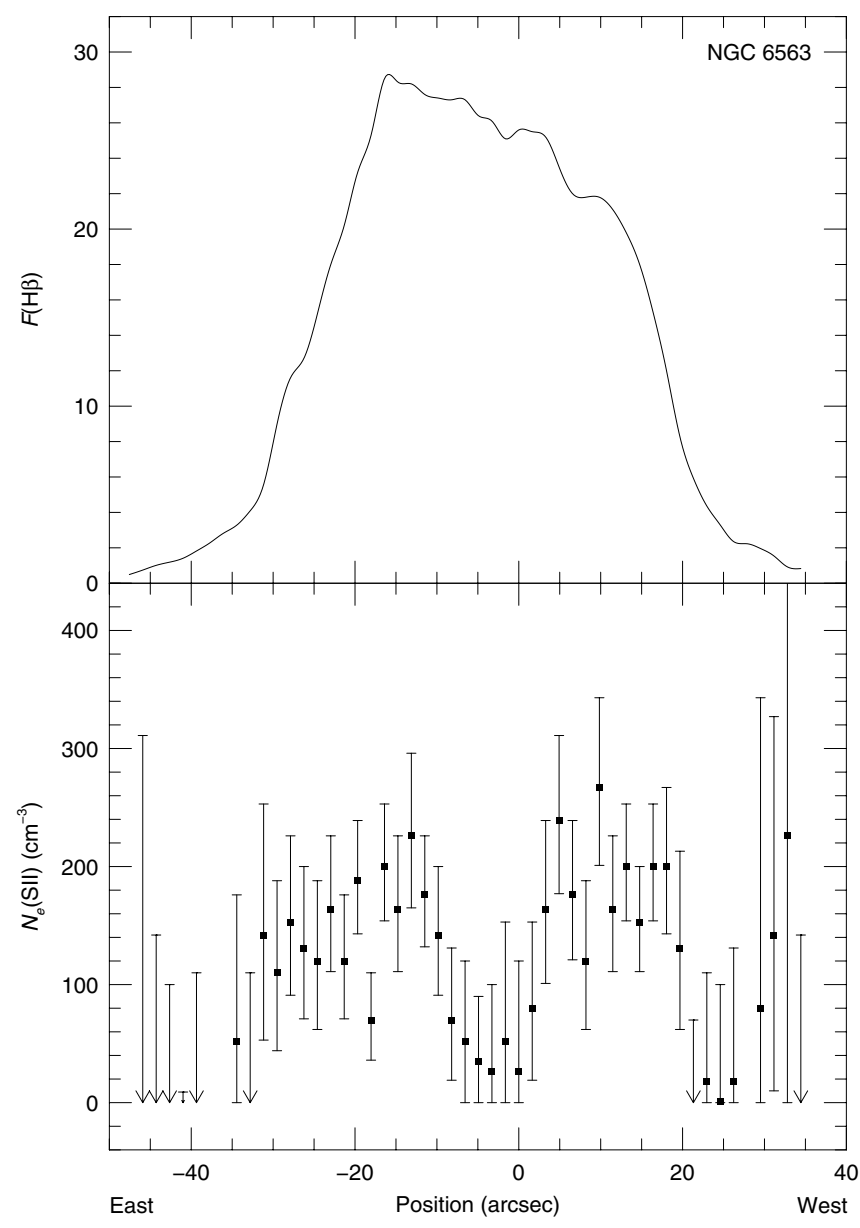

Fig. 16. NGC 6563. Spatial profiles of $\mathrm{H} \beta$ flux (in units of $10^{-15} \mathrm{erg}$ $\left.\mathrm{cm}^{-2} \mathrm{~s}^{-1}\right)$ and $N_{\mathrm{e}}(\mathrm{S} \mathrm{II})$. 
A. C. Krabbe and M. V. F. Copetti: Electron temperature fluctuations, Online Material $p 8$

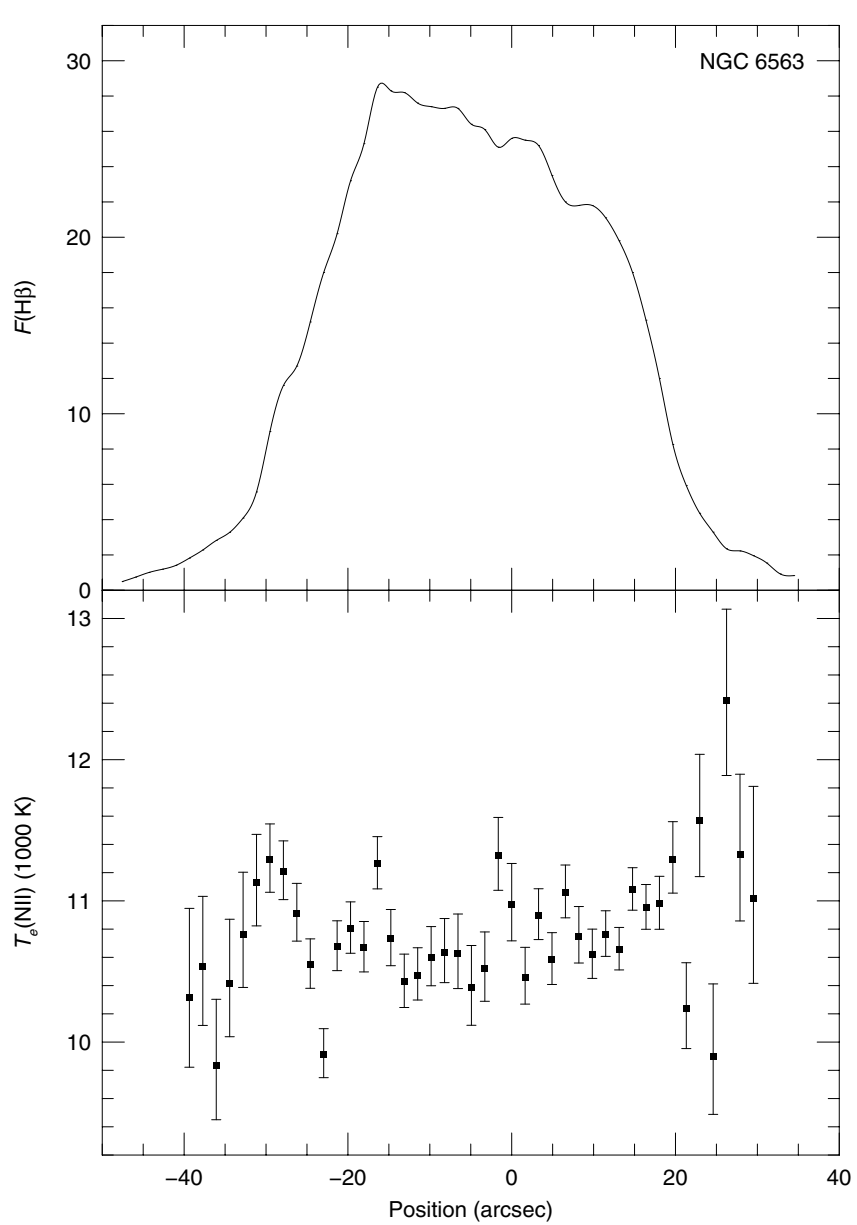

Fig. 17. NGC 6563. Spatial profiles of $\mathrm{H} \beta$ flux (in units of $10^{-15} \mathrm{erg}$ $\left.\mathrm{cm}^{-2} \mathrm{~s}^{-1}\right)$ and $T_{\mathrm{e}}(\mathrm{N} \mathrm{II})$.

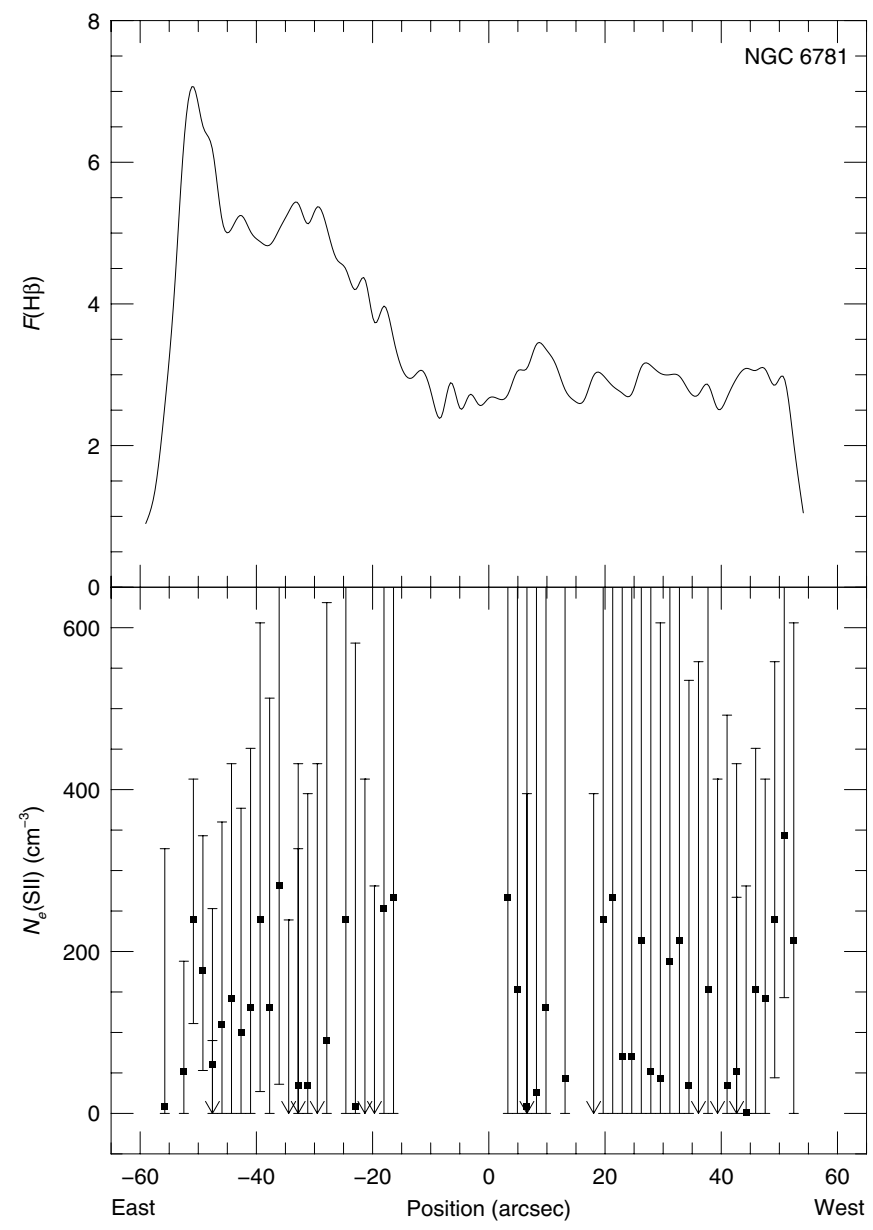

Fig. 18. NGC 6781. Spatial profiles of $\mathrm{H} \beta$ flux (in units of $10^{-15} \mathrm{erg}$ $\left.\mathrm{cm}^{-2} \mathrm{~s}^{-1}\right)$ and $N_{\mathrm{e}}(\mathrm{S} \mathrm{II})$. 
A. C. Krabbe and M. V. F. Copetti: Electron temperature fluctuations, Online Material $p 9$

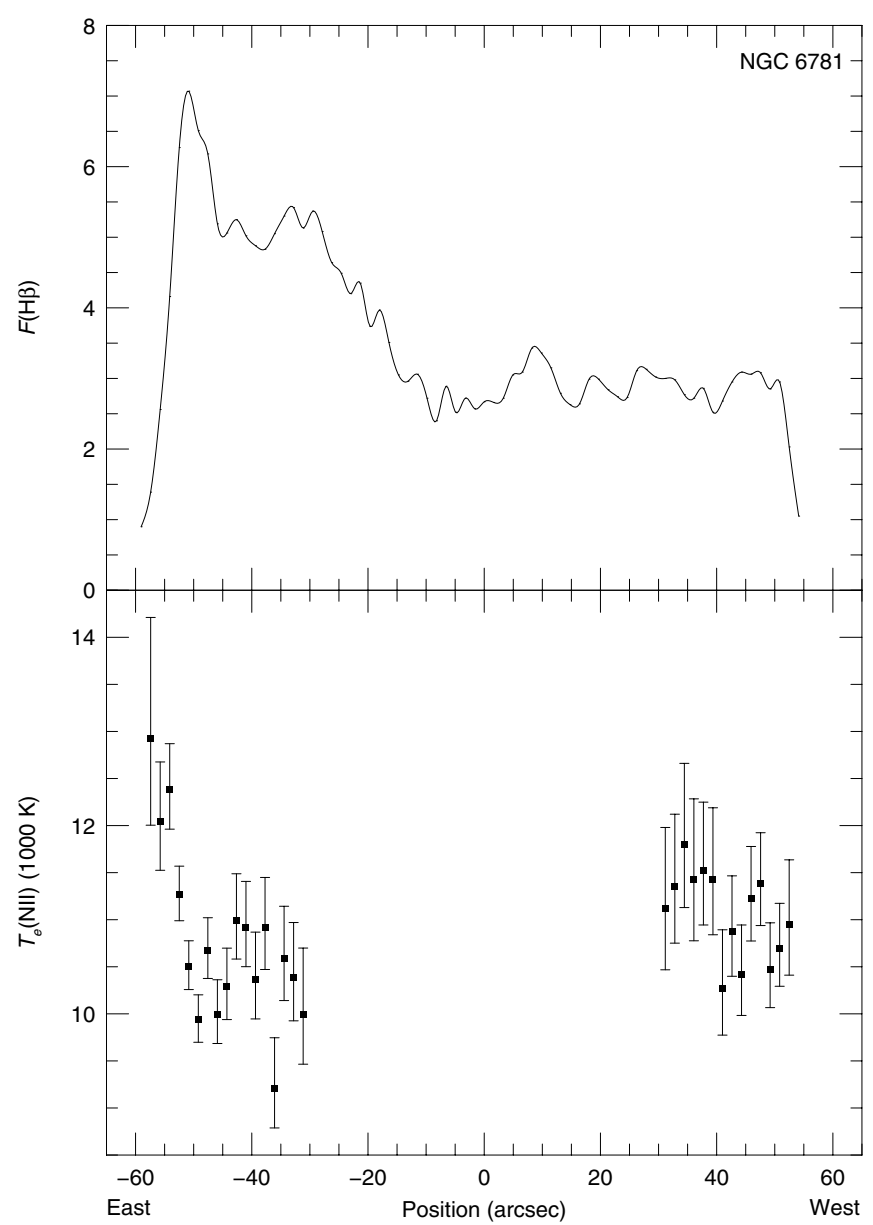

Fig. 19. NGC 6781. Spatial profiles of $\mathrm{H} \beta$ flux (in units of $10^{-15} \mathrm{erg}$ $\left.\mathrm{cm}^{-2} \mathrm{~s}^{-1}\right)$ and $T_{\mathrm{e}}(\mathrm{N} \mathrm{II})$.

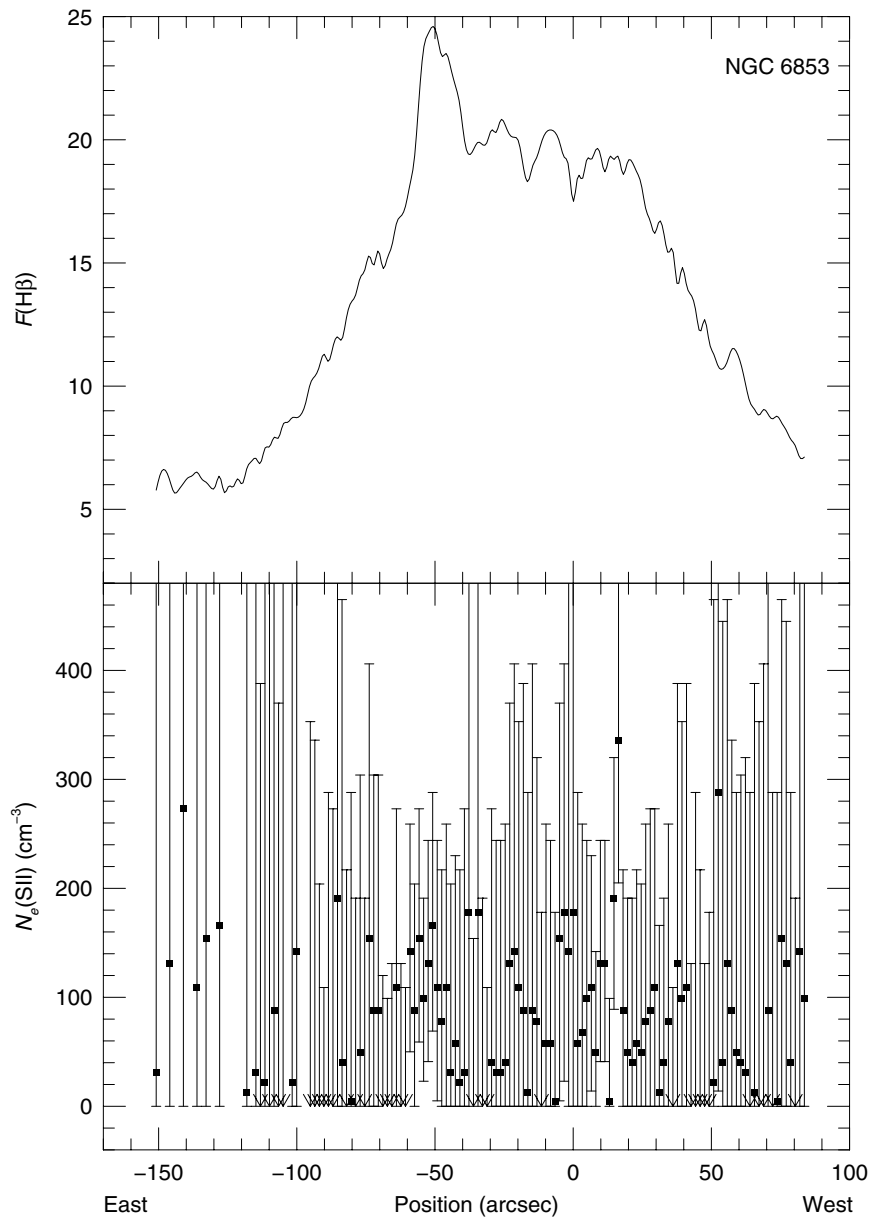

Fig. 20. NGC 6853. Spatial profiles of $\mathrm{H} \beta$ flux (in units of $10^{-15} \mathrm{erg}$ $\mathrm{cm}^{-2} \mathrm{~s}^{-1}$ ) and $N_{\mathrm{e}}(\mathrm{S} \mathrm{II})$. 
A. C. Krabbe and M. V. F. Copetti: Electron temperature fluctuations, Online Material p 10

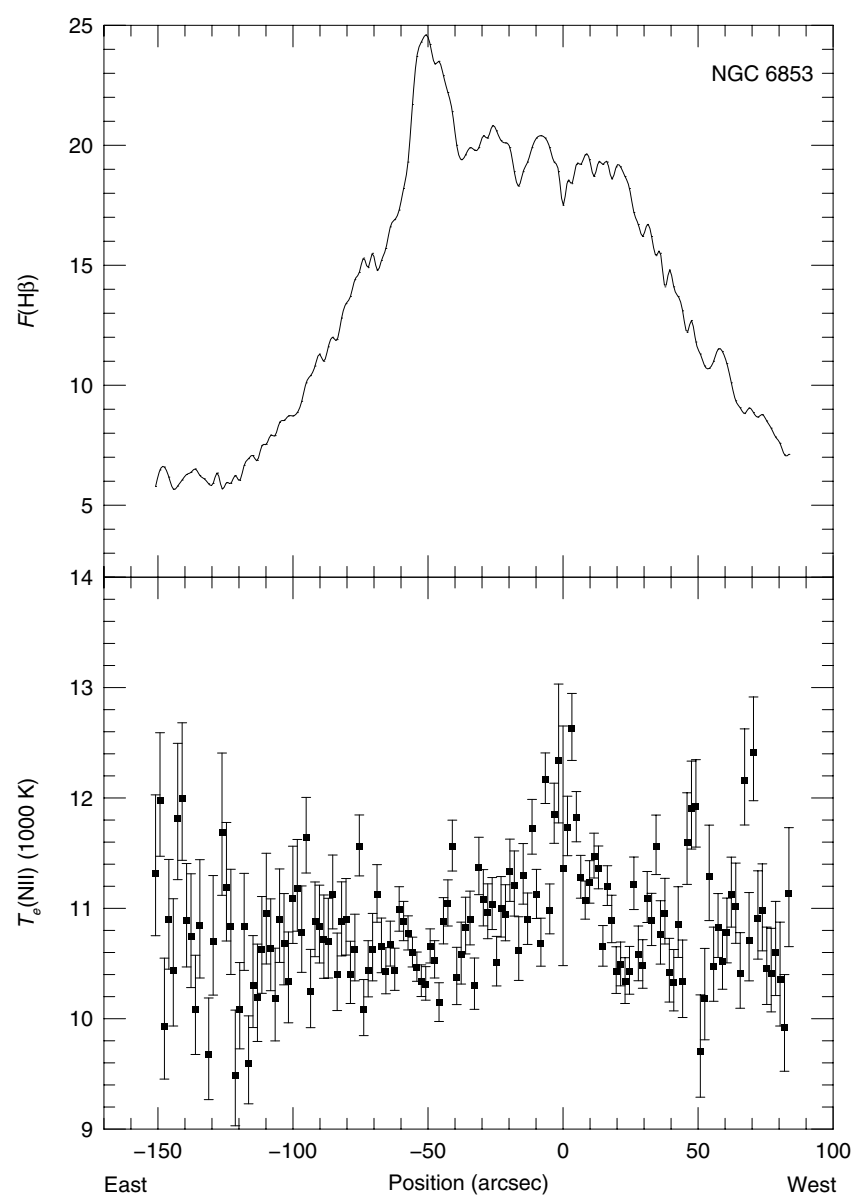

Fig. 21. NGC 6853. Spatial profiles of $\mathrm{H} \beta$ flux (in units of $10^{-15} \mathrm{erg}$ $\left.\mathrm{cm}^{-2} \mathrm{~s}^{-1}\right)$ and $T_{\mathrm{e}}(\mathrm{N} \mathrm{II})$.

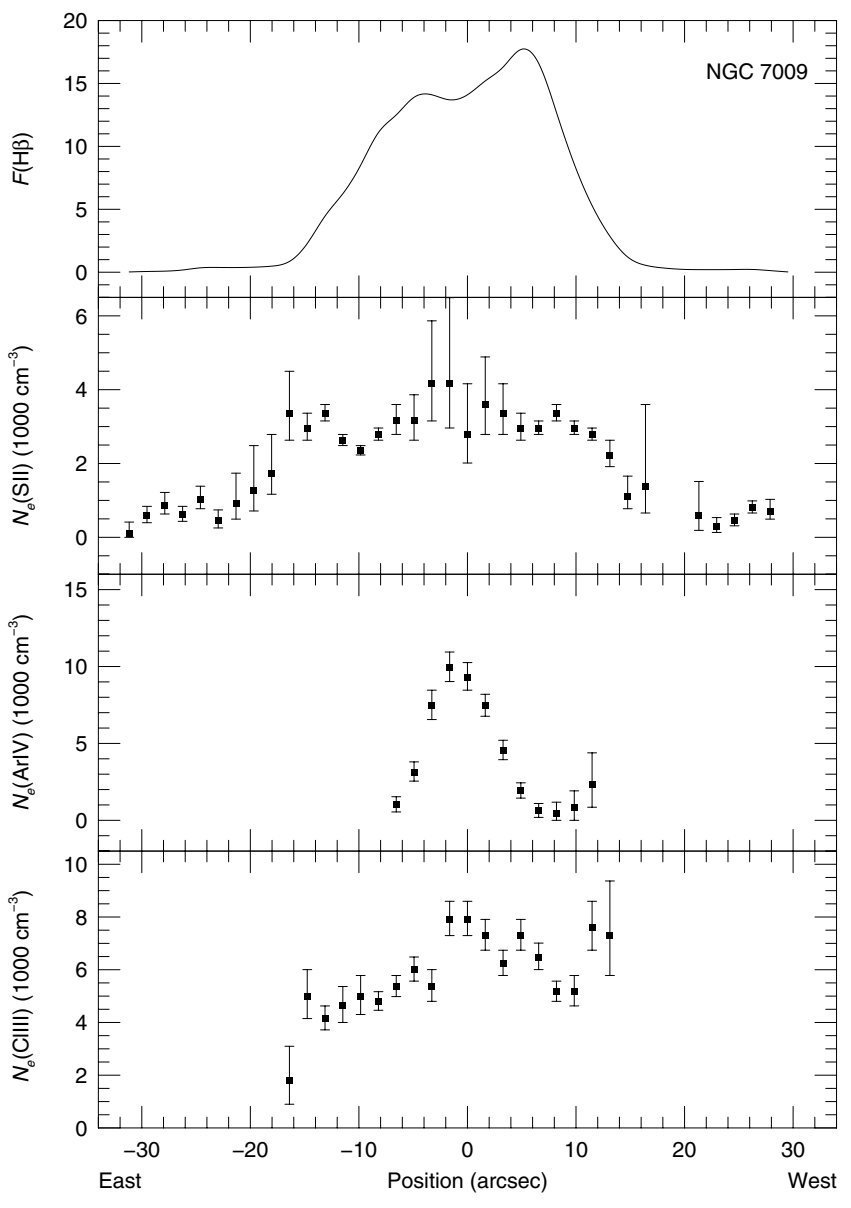

Fig. 22. NGC 7009. Spatial profiles of $\mathrm{H} \beta$ flux (in units of $10^{-13}$ erg $\left.\mathrm{cm}^{-2} \mathrm{~s}^{-1}\right)$ and $N_{\mathrm{e}}(\mathrm{S} \mathrm{II}), N_{\mathrm{e}}(\mathrm{Ar} \mathrm{IV})$ and $N_{\mathrm{e}}(\mathrm{S} \mathrm{II})$. 
A. C. Krabbe and M. V. F. Copetti: Electron temperature fluctuations, Online Material p 11

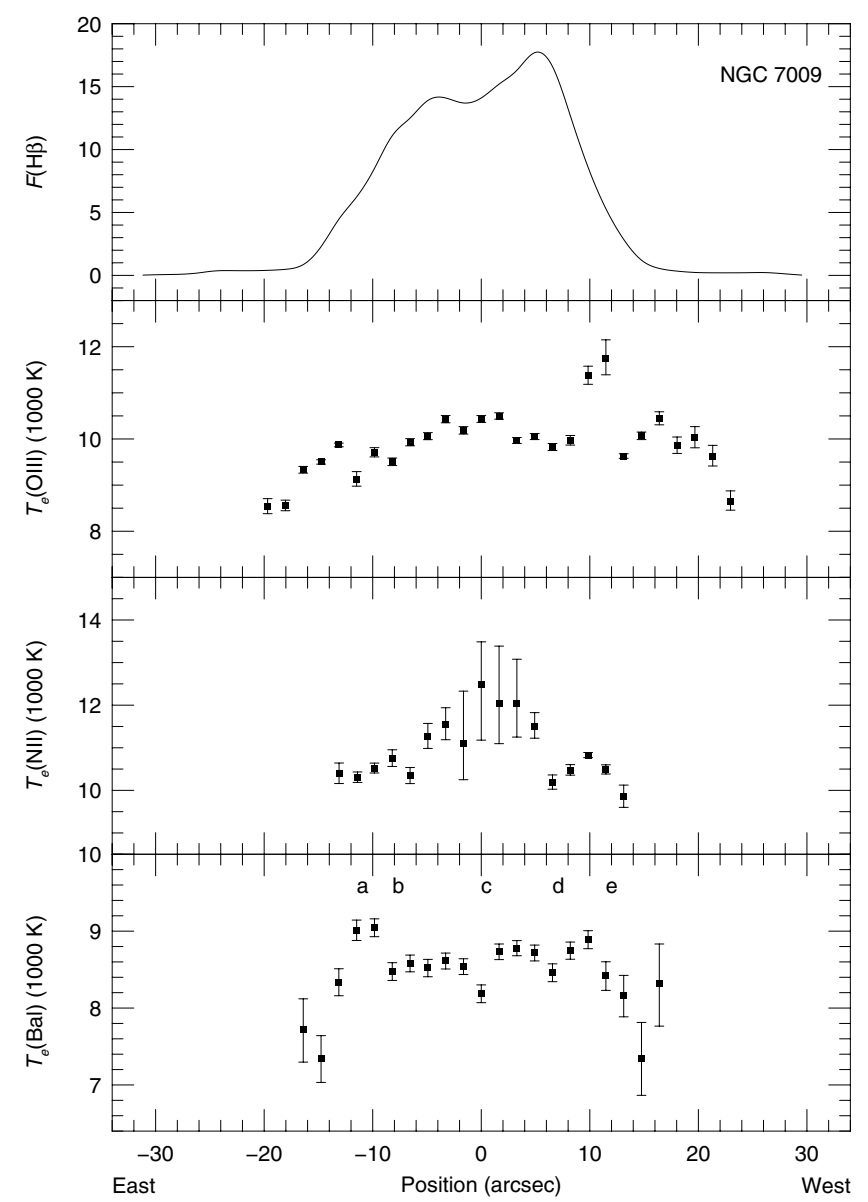

Fig. 23. NGC 7009. Spatial profiles of $\mathrm{H} \beta$ flux (in units of $10^{-13}$ $\left.\mathrm{erg} \mathrm{cm}{ }^{-2} \mathrm{~s}^{-1}\right), T_{\mathrm{e}}(\mathrm{O}$ III $), T_{\mathrm{e}}(\mathrm{N}$ II $)$ and $T_{\mathrm{e}}(\mathrm{Bal})$. 